\title{
Periodic pulse control of Hopf bifurcation in a fractional-order delay predator-prey model incorporating a prey refuge
}

Xiuduo Liu' and Hui Fang ${ }^{1,2^{*}}$

"Correspondence:

mathfanghui@sina.com ${ }^{1}$ Department of Mathematics, Faculty of Science, Kunming University of Science and Technology, Kunming, P.R. China ${ }^{2}$ Center for Nonlinear Science Studies, Kunming University of Science and Technology, Kunming, P.R. China

\section{Springer}

\begin{abstract}
This paper is concerned with periodic pulse control of Hopf bifurcation for a fractional-order delay predator-prey model incorporating a prey refuge. The existence and uniqueness of a solution for such system is studied. Taking the time delay as the bifurcation parameter, critical values of the time delay for the emergence of Hopf bifurcation are determined. A novel periodic pulse delay feedback controller is introduced into the first equation of an uncontrolled system to successfully control the delay-deduced Hopf bifurcation of such a system. Since the stability theory is not well-developed for nonlinear fractional-order non-autonomous systems with delays, we investigate the periodic pulse control problem of the original system by a semi-analytical and semi-numerical method. Specifically, the stability of the linearized averaging system of the controlled system is first investigated, and then it is shown by numerical simulations that the controlled system has the same stability characteristics as its linearized averaging system. The proposed periodic pulse delay feedback controller has more flexibility than a classical linear delay feedback controller guaranteeing the control effect, due to the fact that the pulse width in each control period can be flexibly selected.
\end{abstract}

Keywords: Fractional-order; Time-delay; Hopf bifurcation; Periodic pulse control; Predator-prey system; Linearized averaging system

\section{Introduction}

Long-range temporal memory exists in many population systems [1-4]. Ecological memory was originally defined as "the capacity of past states or experiences to influence present or future responses of the community" [5], and as "the degree to which an ecological process is shaped by its past modifications of a landscape" [6]. Since the Caputo fractional derivative of $q$-order for the function $f \in C^{n}\left(\left[t_{0}, \infty\right), \mathbb{R}\right)\left(n-1<q \leq n \in Z^{+}\right)$at time $t$ depends on the total effect of the usual $n$ th-order integer derivative on the interval $\left[t_{0}, t\right][7$, 8], a fractional-order derivative is associated with whole domain for a biological process [9]. Due to this memory effect, fractional-order systems with Caputo fractional derivatives have more advantages than classical integer-order systems in characterizing the temporal memory. In addition, the fractional theory appearing in various fields of viscoelasticity and electrical engineering, rheology, electrochemistry, biophysics, biology and bioengineering, mechanics, signal and image processing, mechatronics, physics, and control the-

(c) The Author(s) 2019. This article is distributed under the terms of the Creative Commons Attribution 4.0 International License (http://creativecommons.org/licenses/by/4.0/), which permits unrestricted use, distribution, and reproduction in any medium, provided you give appropriate credit to the original author(s) and the source, provide a link to the Creative Commons license, and indicate if changes were made. 
ory have been proposed and studied extensively because of their universal existence and applicability [10]. Recently, Yang et al. proposed new and interesting fractional derivatives without singular kernel [11, 12], and analytic and computational methods for solving nonlinear fractional-order partial differential equations [13, 14], which can be effectively used in the modeling of the fractional-order heat flow $[15,16]$. In the last decade, the dynamics of fractional-order prey-predator systems, such as stability, bifurcations, chaos, have been researched by many investigators [17-26]. In particular, Li et al. [19] investigated the global asymptotic stability of predator-extinction equilibrium point and coexistence equilibrium point for a fractional-order predator-prey model incorporating a prey refuge. Panja [17] studied the stability and dynamics of a three-species predator-prey model with prey, middle predator and top predator. Panja [20] also discussed the uniqueness, boundedness and non-negativity of solutions and the stability around equilibrium points of a fractional-order predator-prey model with intraguild predation.

Due to its complexity and diversity, many systems, such as communication systems, power systems, biological systems, network transmission systems, always inevitably show hysteresis: the rate of change of the current state is not only related to the state of the current moment, but it also depends on the state of a certain moment or a certain period of time in the past. This property of the systems is called time delay, and researchers have introduced differential equations with time delay to describe and study the time-delay system. Especially, the dynamics of predator-prey (PP) systems with delay have been proposed and studied extensively. Many researchers have considered the impact of the past states of biological systems on the present and the future, i.e. incorporating time delay into biological models to describe resource regeneration time, maturation time, reaction time, capturing time, feeding time, gestation period [27, 28]. On the other hand, time-delay biological systems have more complex and richer dynamic behaviors: delays can cause the loss of stability and can induce periodic solutions (Hopf bifurcation), chaos and various oscillations [29-34].

In order to be more consistent with the actual development of biological populations, many researchers combine the fractional-order derivative with the time delay in the model to describe and investigate different complex systems, such as predator-prey interactions with memory effect [35-38], and fractional-order neural networks with delays [39, 40].

Based on the above analysis, we will add a time delay into the model proposed by Li et al. [19], and consider a fractional-order delay predator-prey model incorporating a prey refuge in this paper.

More recently, Hopf bifurcations for delayed fractional-order system have received growing attention. However, it should be noted that researchers pay little attention to the control of bifurcation. Time-delayed feedback is one of the most important regulatory mechanisms in biology. Pyragas [41] initially proposed the delayed feedback control (DFC) in nonlinear autonomous differential equations as a method for stabilizing unstable periodic orbits by using the difference between the current state and the delayed state, that is, $K(y(t)-y(t-\tau))$, where $K$ is called the feedback gain. Bifurcation dynamics for a nonlinear system can be reduced or suppressed by designing an appropriate controller, thereby achieving some desirable dynamical behaviors $[42,43]$. Recently, several novel controllers were introduced for the bifurcation control problem of some delayed fractional predatorprey systems [44-46] and delayed fractional-order network models [47-49]. Huang et al. $[44,45]$ introduced a linear delayed feedback controller and a generalized delayed feed- 
back controller, respectively, to successfully control the Hopf bifurcation in a delayed fractional predator-prey system with incommensurate orders, and it is further certified that the occurrence of the bifurcation can be delayed as feedback gain decreases.

However, to the best of our knowledge, there are few papers discussing the issue of periodic pulse controller in fractional biological population models. Wang et al. [50] introduced a periodic pulse control for Chua's circuit. Considering the periodic changing of environment (e.g., seasonal effects of weather, food supplies and economic reasons, etc.), periodic pulse control is an effective control strategy for the infrequent control. In order to reduce the control time costs and to facilitate implementation, we also introduce a periodic pulse controller in our proposed fractional-order delay predator-prey model incorporating a prey refuge.

The main contributions of this paper are as follows. First, the existence and uniqueness of the solution for our proposed system is studied by using the Banach contraction principle. Second, Hopf bifurcation is studied by taking the time delay as the bifurcation parameter, and sufficient conditions for the characteristic equation has at least one pair of pure imaginary roots and the emergence of Hopf bifurcation are established. Finally, a periodic pulse delay feedback controller is introduced into the first equation of the original system to control the Hopf bifurcation successfully. The biological interpretations of main theoretical results are also given.

The rest of this paper is organized as follows. In Sect. 2, Mathematical model is formulated. In Sect. 3, some definitions are given. In Sect. 4, the existence and uniqueness of the solution for our proposed system was studied, Hopf bifurcation of uncontrolled delayed fractional-order predator-prey model incorporating a prey refuge is analyzed, and a periodic pulse delay feedback controller is designed to control the Hopf bifurcation for the proposed model. In Sect. 5, numerical simulations are carried out to verify our theoretical findings. The conclusions and discussions are given in Sect. 6. Finally, sample Matlab codes for the graphical solutions are listed in the appendices.

\section{Model formulation}

Li et al. [19] investigated the following fractional-order predator-prey model incorporating a prey refuge:

$$
\left\{\begin{array}{l}
{ }_{t_{0}} \mathcal{D}_{t}^{q} x(t)=r x(t)\left(1-\frac{x(t)}{k}\right)-c(1-m) x(t) y(t), \quad x\left(t_{0}\right)=x_{t_{0}}, \\
c_{t_{0}} \mathcal{D}_{t}^{q} y(t)=e c(1-m) x(t) y(t)-d y(t), \quad y\left(t_{0}\right)=y_{t_{0}},
\end{array}\right.
$$

where $0<q<1,{ }_{t_{0}}^{c} \mathcal{D}_{t}^{q}$ denotes the Caputo fractional derivative and $t_{0} \geq 0$ denotes the initial time; $x(t)$ and $y(t)$ denote the densities of prey and predator populations at time $t$, respectively, and all constants $r, k, c, d, e$ are positive, $m \in[0,1)$ is constant. For more detailed description of the model, we refer to [19]. The dynamics of the prey-predator systems incorporating a prey refuge are immensely valuable due to its realism, i.e. for a number of prey populations there are some forms of refuge where they are safe and therefore the prey species can be prevented well from extinction.

It is more realistic to consider the maturation time of the predator in the model (2.1). In this paper, we consider the following fractional-order predator-prey model with time 
delay:

$$
\left\{\begin{array}{l}
{ }_{t_{0}}^{c} \mathcal{D}_{t}^{q} x(t)=r x(t)\left(1-\frac{x(t)}{k}\right)-c(1-m) x(t) y(t-\tau), \\
{ }_{t_{0}}^{c} \mathcal{D}_{t}^{q} y(t)=e c(1-m) x(t) y(t-\tau)-d y(t),
\end{array}\right.
$$

where $\tau>0$ is regarded as the maturation time of the predator, other parameters $r, k, c$, $m, e, d$ have the same meaning as system (2.1), and initial conditions $x(0)>0$ and $y(t)=$ $\phi(t)>0(t \in[-\tau, 0])$, where $\phi(t)$ is a smooth function. We will study the impact of the time delay $\tau$ on the dynamics of the model (2.2).

Ecological control refers to the process of artificially interfering with the ecosystem to make it dynamic. Compared with the real-time control, the periodic pulse control can intermittently control the ecosystem according to periodic factors such as seasonal and weather changes under guaranteeing the control stability effect. This periodically intermittent control can fully exert and stimulate the self-regulation and self-recovery of the ecosystem, which can improve its ability to cope with external intervention and destruction. It is of high biological importance for introducing periodic pulse delay feedback controllers in ecosystems, since periodic pulse controls can enhance the long-term stable existence and development of ecosystems, thereby enriching species diversity.

This paper is concerned with periodic pulse control problem of Hopf bifurcation of system (2.2). In order to reduce the control time costs and facilitate implementation in practice, we introduce a periodic pulse delay feedback controller of the form $\delta(x(t)-x(t-$ $\tau)) P_{T, p}(t)$, where

$$
P_{T, p}(t)=\frac{T}{p} \sum_{n=0}^{+\infty} F_{n, T, p}(t)
$$

and

$$
F_{n, T, p}(t)= \begin{cases}1, & n T \leq t<n T+p, \\ 0, & \text { elsewhere. }\end{cases}
$$

$P_{T, p}(t)$ is a periodic pulse train with a pulse width of $0<p \leq T$ and a period of $T>0$ [50]. It is easy to see that, when $p=T$, a periodic pulse delay feedback controller $\delta(x(t)-x(t-$ $\tau)) P_{T, p}(t)$ can be reduced to a linear delay feedback controller $\delta(x(t)-x(t-\tau))$. Indeed, we impose a linear control of the system during the time interval $n T \leq t<n T+p$, and we do not intervene the system during the time interval $n T+p \leq t<(n+1) T$. In practical problems, we can adjust the size of $p$ to realize expected effects. Thereby periodic pulse control has more flexibility than classical linear control.

\section{Preliminaries}

In this section, we will introduce some fundamental definitions.

Definition $1([7,8])$ The Riemann-Liouville fractional integral operator of order $q$ of a continuous function $f:\left[t_{0}, \infty\right) \rightarrow \mathbb{R}$ is defined as

$$
I^{q} f(t)=\frac{1}{\Gamma(q)} \int_{0}^{t}(t-\zeta)^{q-1} f(\zeta) d \zeta
$$

where $\Gamma(\cdot)$ is the Gamma function, $q>0$. 
Definition 2 ( $[7,8])$ The Caputo fractional derivative of order $q$ of a function $f \in$ $C^{n}\left(\left[t_{0}, \infty\right), \mathbb{R}\right)$ is defined by

$$
{ }_{t_{0}}^{c} \mathcal{D}_{t}^{q} f(t)=\frac{1}{\Gamma(n-q)} \int_{t_{0}}^{t}(t-\zeta)^{n-q-1} f^{(n)}(\zeta) d \zeta,
$$

where $n$ is a positive integer such that $n-1<q \leq n$. In particular, when $0<q \leq 1$, we have

$$
{ }_{t_{0}}^{c} \mathcal{D}_{t}^{q} f(t)=\frac{1}{\Gamma(1-q)} \int_{t_{0}}^{t} \frac{f^{\prime}(\zeta)}{(t-\zeta)^{q}} d \zeta .
$$

Definition $3([7,8])$ The Laplace transform of the Caputo fractional derivative of order $n-1<q \leq n$ for a function $f \in C^{n}\left(\left[t_{0}, \infty\right), \mathbb{R}\right)$ is

$$
L\left\{{ }_{t_{0}}^{c} \mathcal{D}_{t}^{q} f(t) ; s\right\}=s^{q} F(s)-\sum_{k=0}^{n-1} s^{q-k-1} f^{(k)}\left(t_{0}\right),
$$

where $F(s)$ is the Laplace transform of $f(t)$, and $f^{(k)}\left(t_{0}\right)(k=0, \ldots, n-1)$ are the initial conditions. Obviously

$$
L\left\{{ }_{t_{0}}^{c} \mathcal{D}_{t}^{q} f(t) ; s\right\}=s^{q} F(s),
$$

if $f^{(k)}\left(t_{0}\right)=0$ for $k=0, \ldots, n-1$.

Lemma 1 ([51]) Consider the following n-dimensional fractional-order system with delay:

$$
{ }_{t_{0}}^{c} \mathcal{D}_{t}^{q} x_{j}(t)=f_{j}\left(x_{1}(t), \ldots, x_{n}(t) ; \tau\right), \quad j=1,2, \ldots, n,
$$

where $0<q<1$ and the time delay $\tau \geq 0$. System (3.1) undergoes a Hopf bifurcation at the equilibrium $x^{*}=\left(x_{1}^{*}, \ldots, x_{n}^{*}\right)$ when $\tau=\tau_{0}$ if the following conditions are satisfied:

(1) All the eigenvalues $\lambda_{j}(j=1,2, \ldots, n)$ of the coefficient matrix $A$ of the linearized system of (3.1) with $\tau=0$ satisfy $\left|\arg \left(\lambda_{j}\right)\right|>\frac{q \pi}{2}$.

(2) The characteristic equation of the linearized system of (3.1) has a pair of purely imaginary roots $\pm i \omega_{0}$ when $\tau=\tau_{0}$.

(3) $\left.\operatorname{Re}\left[\frac{d s(\tau)}{d \tau}\right]\right|_{\left(\tau=\tau_{0}, \omega=\omega_{0}\right)}>0$, where $\operatorname{Re}[\cdot]$ denotes the real part of the complex number.

\section{Main results}

In this section, we first investigate the existence and uniqueness of a solution for system (2.2). Next, we address the Hopf bifurcation of system (2.2) by taking the time delay $\tau$ as the bifurcation parameter. Last, a periodic pulse delay feedback controller $\delta[x(t)-x(t-$ $\tau)] P_{T, p}(t)$ is introduced into the first equation of an uncontrolled system (2.2) to control the Hopf bifurcation of such a system.

\subsection{Existence and uniqueness}

We study the existence and uniqueness of a solution for the initial value problem

$$
\begin{aligned}
& { }_{t_{0}}^{c} \mathcal{D}_{t}^{q} x(t)=r x(t)\left(1-\frac{x(t)}{k}\right)-c(1-m) x(t) y(t-\tau), \\
& { }_{t_{0}}^{c} \mathcal{D}_{t}^{q} y(t)=e c(1-m) x(t) y(t-\tau)-d y(t), \quad t \in\left[t_{0}, t_{0}+H\right], \\
& (x(t), y(t))=\psi(t):=\left(\psi_{1}(t), \psi_{2}(t)\right), \quad t \in\left[t_{0}-\tau, t_{0}\right],
\end{aligned}
$$


where $0<q \leq 1, t_{0} \geq 0, \tau>0, H>0$, and the initial value function $\psi(t) \in C\left(\left[t_{0}-\tau, t_{0}\right]\right.$, $\mathbb{R}^{2}$ ).

\section{Denote}

$$
X(t)=(x(t), y(t)), \quad f(X(t))=\left(f_{1}(X(t)), f_{2}(X(t)),\right.
$$

where

$$
\begin{aligned}
& f_{1}(X(t))=r x(t)\left(1-\frac{x(t)}{k}\right)-c(1-m) x(t) y(t-\tau), \\
& f_{2}(X(t))=e c(1-m) x(t) y(t-\tau)-d y(t) .
\end{aligned}
$$

For $X=(x, y) \in \mathbb{R}^{2}$, take the norm $\|X\|=|x|+|y|$. Take $X=C\left(\left[t_{0}-\tau, t_{0}+H\right], \mathbb{R}^{2}\right)$, and define the norm $\|X\|_{X}=\max _{t \in\left[t_{0}-\tau, t_{0}+H\right]}\|X(t)\|$ for $X(t)=(x(t), y(t)) \in \mathcal{X}$.

Set

$$
\mathscr{D}=\left\{X \in \mathcal{X}: X(t)=\psi(t) \text { for } t \in\left[t_{0}-\tau, t_{0}\right] \text {, and } \max _{t \in\left[t_{0}, t_{0}+H\right]}\left\|X(t)-\psi\left(t_{0}\right)\right\| \leq G\right\}
$$

$(G>0)$.

Clearly, for any $X(t) \in \mathcal{D}$, we have $\|X\|_{X} \leq M:=\max \left\{\max _{t \in\left[t_{0}-\tau, t_{0}\right]}\|\psi(t)\|,\left\|\psi\left(t_{0}\right)\right\|+G\right\}$. Therefore, for any $X(t)=(x(t), y(t)), \bar{X}(t)=(\bar{x}(t), \bar{y}(t)) \in \mathscr{D}, t \in\left[t_{0}, t_{0}+H\right]$, we have

$$
\begin{aligned}
&\|f(X(t))-f(\bar{X}(t))\| \\
&=\left|f_{1}(X(t))-f_{1}(\bar{X}(t))\right|+\left|f_{2}(X(t))-f_{2}(\bar{X}(t))\right| \\
&=\left|r x(t)\left(1-\frac{x(t)}{k}\right)-c(1-m) x(t) y(t-\tau)-r \bar{x}(t)\left(1-\frac{\bar{x}(t)}{k}\right)+c(1-m) \bar{x}(t) \bar{y}(t-\tau)\right| \\
& \quad+|e c(1-m) x(t) y(t-\tau)-d y(t)-e c(1-m) \bar{x}(t) \bar{y}(t-\tau)+d \bar{y}(t)| \\
&=\mid r(x(t)-\bar{x}(t))-\frac{r}{k}(x(t)+\bar{x}(t))(x(t)-\bar{x}(t))-c(1-m) y(t-\tau)(x(t)-\bar{x}(t)) \\
&-c(1-m) \bar{x}(t)(y(t-\tau)-\bar{y}(t-\tau))|+| e c(1-m) y(t-\tau)(x(t)-\bar{x}(t)) \\
&+e c(1-m) \bar{x}(t)(y(t-\tau)-\bar{y}(t-\tau))-d(y(t)-\bar{y}(t)) \mid \\
& \leq\left|r-\frac{r}{k}(x(t)+\bar{x}(t))-c(1-m) y(t-\tau)\right| \cdot|x(t)-\bar{x}(t)| \\
&+|c(1-m) \bar{x}(t)| \cdot|y(t-\tau)-\bar{y}(t-\tau)|+|e c(1-m) y(t-\tau)| \cdot|x(t)-\bar{x}(t)| \\
&+|e c(1-m) \bar{x}(t)| \cdot|y(t-\tau)-\bar{y}(t-\tau)|+d|y(t)-\bar{y}(t)| \\
& \leq\left(r+\frac{2 r M}{k}+c(1+e)(1-m) M\right)|x(t)-\bar{x}(t)|+d|y(t)-\bar{y}(t)| \\
&+c(1+e)(1-m) M|y(t-\tau)-\bar{y}(t-\tau)| \\
& \leq L(\|X(t)-\bar{X}(t)\|+\|X(t-\tau)-\bar{X}(t-\tau)\|),
\end{aligned}
$$

where $L:=\max \left\{r+\frac{2 r M}{k}+c(1+e)(1-m) M, d\right\}$. 
Similarly, for any $X(t) \in \mathcal{D}, t \in\left[t_{0}, t_{0}+H\right]$, we have

$$
\begin{aligned}
\|f(X(t))\|= & \left|f_{1}(X(t))\right|+\left|f_{2}(X(t))\right| \\
= & \left|r x(t)\left(1-\frac{x(t)}{k}\right)-c(1-m) x(t) y(t-\tau)\right| \\
& +|e c(1-m) x(t) y(t-\tau)-d y(t)| \\
\leq & \left(r+\frac{r|x(t)|}{k}+c(1+e)(1-m)|y(t-\tau)|\right)|x(t)|+d|y(t)| \\
\leq & \left(r+\frac{r M}{k}+c(1+e)(1-m) M\right)|x(t)|+d|y(t)| \\
\leq & L\|X(t)\| .
\end{aligned}
$$

Next, by applying the fractional integral operator to system (4.1), (4.1) can be transformed into the following equivalent Volterra equation of the second kind:

$$
\begin{aligned}
& X(t)=\psi\left(t_{0}\right)+\frac{1}{\Gamma(q)} \int_{t_{0}}^{t}(t-s)^{q-1} f(X(s)) d s, \quad t \in\left[t_{0}, t_{0}+H\right] \\
& X(t)=\psi(t)=\left(\psi_{1}(t), \psi_{2}(t)\right), \quad t \in\left[t_{0}-\tau, t_{0}\right] .
\end{aligned}
$$

Define the operator $\mathcal{P}: \mathscr{D} \rightarrow \mathscr{D}$, such that

$$
\begin{aligned}
& \mathcal{P} X(t):=\psi\left(t_{0}\right)+\frac{1}{\Gamma(q)} \int_{t_{0}}^{t}(t-s)^{q-1} f(X(s)) d s, \quad t \in\left[t_{0}, t_{0}+H\right] \\
& \mathcal{P} X(t):=\psi(t)=\left(\psi_{1}(t), \psi_{2}(t)\right), \quad t \in\left[t_{0}-\tau, t_{0}\right] .
\end{aligned}
$$

Then $\mathcal{P}$ has a unique fixed point in $\mathscr{D}$ implies that problem (4.1) has a unique solution.

By (4.3) and (4.5), for any $X(t)=(x(t), y(t)), \bar{X}(t)=(\bar{x}(t), \bar{y}(t)) \in \mathcal{D}, t \in\left[t_{0}, t_{0}+H\right]$, we have

$$
\begin{aligned}
&\|\mathcal{P}(X(t))-\mathcal{P}(\bar{X}(t))\| \\
& \leq \frac{1}{\Gamma(q)} \int_{t_{0}}^{t}(t-s)^{q-1}\|f(X(s))-f(\bar{X}(s))\| d s \\
& \leq \frac{L}{\Gamma(q)} \int_{t_{0}}^{t}(t-s)^{q-1}(\|X(s)-\bar{X}(s)\|+\|X(s-\tau)-\bar{X}(s-\tau)\|) d s \\
& \leq \frac{L}{\Gamma(q)} \int_{t_{0}}^{t}(t-s)^{q-1}\left(\max _{s \in\left[t_{0}, t_{0}+H\right]}\|X(s)-\bar{X}(s)\|\right. \\
&\left.\quad+\max \left\{_{s \in\left[t_{0}-\tau, t_{0}\right]}\|X(s)-\bar{X}(s)\|, \max _{s \in\left[t_{0}, t_{0}+H\right]}\|X(s)-\bar{X}(s)\|\right\}\right) d s \\
& \leq \frac{2 L}{\Gamma(q)} \int_{t_{0}}^{t}(t-s)^{q-1}\left(\max _{s \in\left[t_{0}, t_{0}+H\right]}\|X(s)-\bar{X}(s)\|\right) d s \\
& \leq \frac{2 L H^{q}}{\Gamma(q+1)}\|X-\bar{X}\|_{X} .
\end{aligned}
$$

So, we have $\|\mathcal{P}(X(\cdot))-\mathcal{P}(\bar{X}(\cdot))\|_{X} \leq \frac{2 L H^{q}}{\Gamma(q+1)}\|X-\bar{X}\|_{X}$, which implies that $\mathcal{P}$ is a contraction operator if $H<\left(\frac{\Gamma(q+1)}{2 L}\right)^{1 / q}$. 
For any $X(t) \in \mathcal{D}, t \in\left[t_{0}, t_{0}+H\right]$, by (4.4) and (4.5), we have

$$
\begin{aligned}
\left\|\mathcal{P}(X(t))-\psi\left(t_{0}\right)\right\| & \leq \frac{1}{\Gamma(q)} \int_{t_{0}}^{t}(t-s)^{q-1}\|f(X(s))\| d s \\
& \leq \frac{L}{\Gamma(q)} \int_{t_{0}}^{t}(t-s)^{q-1}\|X(s)\| d s \\
& \leq \frac{L}{\Gamma(q)} \int_{t_{0}}^{t}(t-s)^{q-1} \max _{s \in\left[t_{0}, t_{0}+H\right]}\|X(s)\| d s \\
& \leq \frac{L H^{q}}{\Gamma(q+1)} \max _{s \in\left[t_{0}, t_{0}+H\right]}\|X(s)\| \\
& \leq \frac{L H^{q} M}{\Gamma(q+1)} .
\end{aligned}
$$

If $H \leq\left(\frac{\Gamma(q+1) G}{L M}\right)^{1 / q}$, then it follows from (4.6) that $\max _{t \in\left[t_{0}, t_{0}+H\right]}\left\|\mathcal{P}(X(t))-\psi\left(t_{0}\right)\right\| \leq G$, which implies $\mathcal{P}(X(t)) \in \mathscr{D}$ for any $X(t) \in \mathscr{D}$.

By the Banach contraction principle, $\mathcal{P}$ has a unique fixed point in $\mathscr{D}$ when $H<$ $\min \left\{\left(\frac{\Gamma(q+1) G}{L M}\right)^{1 / q},\left(\frac{\Gamma(q+1)}{2 L}\right)^{1 / q}\right\}$. From the above analysis, the following theorem can be obtained.

Theorem 1 If $H<\min \left\{\left(\frac{\Gamma(q+1) G}{L M}\right)^{1 / q},\left(\frac{\Gamma(q+1)}{2 L}\right)^{1 / q}\right\}$, then the initial value problem (4.1) has a unique solution.

\subsection{Bifurcation analysis of the uncontrolled system}

For convenience, we introduce the basic reproduction number of the integer-order counterpart of system (2.1). The integer-order counterpart of system (2.1) can be written as

$$
\left\{\begin{array}{l}
\frac{d x(t)}{d t}=r x(t)\left(1-\frac{x(t)}{k}\right)-c(1-m) x(t) y(t), \\
\frac{d y(t)}{d t}=e c(1-m) x(t) y(t)-d y(t)
\end{array}\right.
$$

Lemma 2 For the integer-order counterpart (4.7) of system (2.1), the basic reproduction number is

$$
R_{0}:=\frac{e c(1-m) k}{d}
$$

Proof The basic reproduction number of system (4.7) can be obtained by the next generation method [52]. Since the procedure of calculation is similar to that of Theorem 3 in [24], we omit the details here.

System (2.2) always has trivial equilibrium point $\varepsilon_{0}=(0,0)$ and predator-free equilibrium $\varepsilon_{1}=(k, 0)$. When $R_{0}>1$, system $(2.2)$ has a unique coexistence equilibrium $\varepsilon_{2}=$ $\left(\frac{k}{R_{0}}, \frac{r e k}{d R_{0}^{2}}\left(R_{0}-1\right)\right)$.

The linearized system of (2.2) at equilibrium point $\left(x^{*}, y^{*}\right)$ is defined as

$$
\left\{\begin{array}{l}
{ }_{t_{0}}^{c} \mathcal{D}_{t}^{q} x(t)=\left(r\left(1-\frac{2 x^{*}}{k}\right)-c(1-m) y^{*}\right)\left(x(t)-x^{*}\right)-c(1-m) x^{*}\left(y(t-\tau)-y^{*}\right), \\
{ }_{t_{0}}^{c} \mathcal{D}_{t}^{q} y(t)=e c(1-m) y^{*}\left(x(t)-x^{*}\right)+e c(1-m) x^{*}\left(y(t-\tau)-y^{*}\right)-d\left(y(t)-y^{*}\right) .
\end{array}\right.
$$


Denote

$$
\left\{\begin{array}{l}
a_{1}=c(1-m) y^{*}-r\left(1-\frac{2 x^{*}}{k}\right) \\
a_{2}=c(1-m) x^{*} \\
a_{3}=-e c(1-m) y^{*} \\
a_{4}=-e c(1-m) x^{*} \\
a_{5}=d
\end{array}\right.
$$

Taking the Laplace transform [53] on both sides of system (4.9) (for simplicity, taking $\left.t_{0}=0\right)$ gives

$$
\left\{\begin{aligned}
s^{q} \hat{X}(s)-s^{q-1} x(0)= & -a_{1} \hat{X}(s)+\frac{a_{1} x^{*}}{s}-a_{2} e^{-s \tau}\left(\hat{Y}(s)+\int_{-\tau}^{0} e^{-s t} \phi(t) d t\right)+\frac{a_{2} y^{*}}{s} \\
s^{q} \hat{Y}(s)-s^{q-1} y(0)= & -a_{3} \hat{X}(s)+\frac{a_{3} x^{*}}{s}-a_{4} e^{-s \tau}\left(\hat{Y}(s)+\int_{-\tau}^{0} e^{-s t} \phi(t) d t\right) \\
& +\frac{a_{4} y^{*}}{s}-a_{5} \hat{Y}(s)+\frac{a_{5} y^{*}}{s}
\end{aligned}\right.
$$

where $\hat{X}(s), \hat{Y}(s)$ are the Laplace transform of $x(t), y(t)$ with $\hat{X}(s)=\mathcal{L}(x(t)), \hat{Y}(s)=\mathcal{L}(y(t))$, $x(0)>0$ and $y(t)=\phi(t)>0(t \in[-\tau, 0])$ are initial values. The system (4.10) can be rewritten as follows:

$$
\Delta(s) \cdot\left(\begin{array}{c}
\hat{X}(s) \\
\hat{Y}(s)
\end{array}\right)=\left(\begin{array}{l}
b_{1}(s) \\
b_{2}(s)
\end{array}\right)
$$

where

$$
\Delta(s)=\left(\begin{array}{cc}
s^{q}+a_{1} & a_{2} e^{-s \tau} \\
a_{3} & s^{q}+a_{4} e^{-s \tau}+a_{5}
\end{array}\right)
$$

and

$$
\left(\begin{array}{l}
b_{1}(s) \\
b_{2}(s)
\end{array}\right)=\left(\begin{array}{c}
s^{q-1} x(0)+\frac{a_{1} x^{*}}{s}-a_{2} e^{-s \tau} \int_{-\tau}^{0} e^{-s t} \phi(t) d t+\frac{a_{2} y^{*}}{s} \\
s^{q-1} y(0)+\frac{a_{3} x^{*}}{s}-a_{4} e^{-s \tau} \int_{-\tau}^{0} e^{-s t} \phi(t) d t+\frac{\left(a_{4}+a_{5}\right) y^{*}}{s}
\end{array}\right) .
$$

$\Delta(s)$ is considered as the characteristic matrix of system (4.9) for simplicity.

When $\tau=0$, the coefficient matrix of system (4.9) is recorded as

$$
A=\left(\begin{array}{cc}
-a_{1} & -a_{2} \\
-a_{3} & -\left(a_{4}+a_{5}\right)
\end{array}\right)
$$

In the following, we look for the conditions of system (2.2) exhibiting a Hopf bifurcation at coexistence equilibrium $\varepsilon_{2}=\left(\frac{k}{R_{0}}, \frac{r e k}{d R_{0}^{2}}\left(R_{0}-1\right)\right)$. 
For the coefficient matrix $A$ at coexistence equilibrium $\varepsilon_{2}=\left(x^{*}, y^{*}\right)=\left(\frac{k}{R_{0}}, \frac{r e k}{d R_{0}^{2}}\left(R_{0}-1\right)\right)$, $a_{i}(i=1,2,3,4,5)$ are determined by

$$
\left\{\begin{array}{l}
a_{1}=c(1-m) y^{*}-r\left(1-\frac{2 x^{*}}{k}\right)=\frac{r}{R_{0}} \\
a_{2}=c(1-m) x^{*}=\frac{d}{e} \\
a_{3}=-e c(1-m) y^{*}=-\frac{e r}{R_{0}}\left(R_{0}-1\right) \\
a_{4}=-e c(1-m) x^{*}=-d \\
a_{5}=d
\end{array}\right.
$$

Then we have

$$
\operatorname{det}(A-\lambda I)=\lambda^{2}+\left(a_{1}+a_{4}+a_{5}\right) \lambda+a_{1}\left(a_{4}+a_{5}\right)-a_{2} a_{3}=0 .
$$

If the following conditions are satisfied:

$$
\left\{\begin{array}{l}
a_{1}+a_{4}+a_{5}=\frac{r}{R_{0}}>0, \\
a_{1}\left(a_{4}+a_{5}\right)-a_{2} a_{3}=\frac{d r}{R_{0}}\left(R_{0}-1\right)>0,
\end{array}\right.
$$

i.e. $R_{0}>1$, then it is easy to check from the Routh-Hurwitz criterion that two eigenvalues of the characteristic equation (4.14) have negative real parts. This implies that two eigenvalues of $A$ satisfy the inequality $\left|\arg \left(\lambda_{j}\right)\right|>\frac{\alpha \pi}{2}(j=1,2)$. Thus, the condition (1) of Hopf bifurcation in Lemma 1 is satisfied for system (2.2).

If $\tau>0$, then the characteristic matrix at coexistence equilibrium $\varepsilon_{2}=\left(x^{*}, y^{*}\right)=$ $\left(\frac{k}{R_{0}}, \frac{r e k}{d R_{0}^{2}}\left(R_{0}-1\right)\right)$ is

$$
\Delta_{2}(s)=\left(\begin{array}{cc}
s^{q}+a_{1} & a_{2} e^{-s \tau} \\
a_{3} & s^{q}+a_{4} e^{-s \tau}+a_{5}
\end{array}\right)
$$

where $a_{1}, \ldots, a_{5}$ are determined as in (4.13). Therefore the characteristic equation at coexistence equilibrium $\varepsilon_{2}=\left(\frac{k}{R_{0}}, \frac{r e k}{d R_{0}^{2}}\left(R_{0}-1\right)\right)$ is

$$
\operatorname{det}\left(\Delta_{2}(s)\right)=s^{2 q}+\left(a_{1}+a_{5}\right) s^{q}+a_{4} e^{-s \tau} s^{q}+\left(a_{1} a_{4}-a_{2} a_{3}\right) e^{-s \tau}+a_{1} a_{5}=0 .
$$

Next, we look for the conditions that guarantee the characteristic equation (4.15) has a pair of pure imaginary roots $s= \pm \omega i(\omega>0)$.

Assume that Eq. (4.15) has a pure imaginary root $s=\omega i(\omega>0)$, substituting $s=\omega i$ into Eq. (4.15) gives

$$
\begin{aligned}
& w^{2 q}(\cos (q \pi)+i \sin (q \pi))+w^{q}\left(a_{1}+a_{5}\right)\left(\cos \left(\frac{q \pi}{2}\right)+i \sin \left(\frac{q \pi}{2}\right)\right) \\
& +w^{q} a_{4}\left(\cos \left(\frac{q \pi}{2}\right)+i \sin \left(\frac{q \pi}{2}\right)\right)(\cos (\omega \tau)-i \sin (\omega \tau)) \\
& +\left(a_{1} a_{4}-a_{2} a_{3}\right)(\cos (\omega \tau)-i \sin (\omega \tau))+a_{1} a_{5}=0 .
\end{aligned}
$$


Separating real and imaginary parts of Eq. (4.16) gives

$$
\begin{aligned}
& w^{2 q} \cos (q \pi)+w^{q}\left(a_{1}+a_{5}\right) \cos \left(\frac{q \pi}{2}\right)+a_{1} a_{5} \\
& =-w^{q} a_{4} \cos \left(\omega \tau-\frac{q \pi}{2}\right)-\left(a_{1} a_{4}-a_{2} a_{3}\right) \cos (\omega \tau), \\
& w^{2 q} \sin (q \pi)+w^{q}\left(a_{1}+a_{5}\right) \sin \left(\frac{q \pi}{2}\right) \\
& =w^{q} a_{4} \sin \left(\omega \tau-\frac{q \pi}{2}\right)+\left(a_{1} a_{4}-a_{2} a_{3}\right) \sin (\omega \tau) .
\end{aligned}
$$

By squaring and adding both sides of (4.17), combining (4.13) we have

$$
\begin{aligned}
& w^{4 q}+w^{3 q} \cdot 2\left(\frac{r}{R_{0}}+d\right) \cos \left(\frac{q \pi}{2}\right)+w^{2 q} \cdot\left[\frac{r^{2}}{R_{0}^{2}}+\frac{2 r d}{R_{0}}(1+\cos (q \pi))\right] \\
& +w^{q} \cdot\left[\frac{2 r d}{R_{0}}\left(\frac{r}{R_{0}}+d\left(R_{0}-1\right)\right) \cos \left(\frac{q \pi}{2}\right)\right]+\frac{r^{2} d^{2}}{R_{0}^{2}}\left[-\left(R_{0}-1\right)\left(R_{0}-3\right)\right]=0 .
\end{aligned}
$$

Since $\cos \left(\frac{q \pi}{2}\right)>0, \omega^{q}>0$, Eq. (4.18) has no real solutions if $1<R_{0}<3$. Thus, if $1<R_{0}<3$, then Eq. (4.15) has no purely imaginary roots for any $\tau>0$.

It is easy to see that Eq. (4.15) has at least a pair of pure imaginary roots if and only if Eq. (4.18) has at least a positive root. Put $v=\omega^{q}$, then Eq. (4.18) can be rewritten as

$$
\begin{aligned}
v^{4} & +2\left(\frac{r}{R_{0}}+d\right) \cos \left(\frac{q \pi}{2}\right) v^{3}+\left[\frac{r^{2}}{R_{0}^{2}}+\frac{2 r d}{R_{0}}(1+\cos (q \pi))\right] v^{2} \\
& +\left[\frac{2 r d}{R_{0}}\left(\frac{r}{R_{0}}+d\left(R_{0}-1\right)\right) \cos \left(\frac{q \pi}{2}\right)\right] v+\frac{r^{2} d^{2}}{R_{0}^{2}}\left[-\left(R_{0}-1\right)\left(R_{0}-3\right)\right]=0 .
\end{aligned}
$$

Then Eq. (4.18) has at least one positive root if and only if Eq. (4.19) has at least one positive root. Write Eq. (4.19) as

$$
v^{4}+p_{1} v^{3}+p_{2} v^{2}+p_{3} v+p_{4}=0
$$

where $p_{i}(i=1,2,3,4)$ are the corresponding coefficient in Eq. (4.19). Denote $h(v):=v^{4}+$ $p_{1} v^{3}+p_{2} v^{2}+p_{3} v+p_{4}$. If $R_{0}>3$, then $p_{4}=\frac{r^{2} d^{2}}{R_{0}^{2}}\left[-\left(R_{0}-1\right)\left(R_{0}-3\right)\right]<0$. Since $h(0)=p_{4}<0$ and $\lim _{v \rightarrow+\infty} h(v)=+\infty$, there exists a $v_{0}>0$ such that $h\left(v_{0}\right)=0$. Finally, we calculate the delay $\tau_{0}$ which guarantees the existence of pure imaginary roots in Eq. (4.15). For simplicity, denote $C_{1}=a_{1}+a_{5}, C_{2}=a_{4}, D_{1}=a_{1} a_{4}-a_{2} a_{3}, D_{2}=a_{1} a_{5}$. Then Eq. (4.15) is equivalent to

$$
s^{2 q}+C_{1} s^{q}+D_{2}+\left(C_{2} s^{q}+D_{1}\right) e^{-s \tau}=0
$$

Set

$$
\begin{aligned}
& A=s^{2 q}+C_{1} s^{q}+D_{2}, \\
& E=C_{2} s^{q}+D_{1} .
\end{aligned}
$$


Then Eq. (4.20) is equivalent to

$$
A+E e^{-s \tau}=0 .
$$

Let $s=\omega i(\omega>0)$ and $A_{i}, E_{i}(i=1,2)$ be the real and imaginary parts of $A, E$, respectively. Thus, Eq. (4.21) can be written as

$$
\left(A_{1}+i A_{2}\right)+\left(E_{1}+i E_{2}\right)(\cos (\omega \tau)-i \sin (\omega \tau))=0,
$$

where

$$
\begin{aligned}
& A_{1}=\omega^{2 q} \cos (q \pi)+C_{1} \omega^{q} \cos \left(\frac{q \pi}{2}\right)+D_{2}, \\
& A_{2}=\omega^{2 q} \sin (q \pi)+C_{1} \omega^{q} \sin \left(\frac{q \pi}{2}\right),
\end{aligned}
$$

and

$$
\begin{aligned}
& E_{1}=C_{2} \omega^{q} \cos \left(\frac{q \pi}{2}\right)+D_{1}, \\
& E_{2}=C_{2} \omega^{q} \sin \left(\frac{q \pi}{2}\right) .
\end{aligned}
$$

Separating the real imaginary parts of Eq. (4.22), one has

$$
\left\{\begin{array}{l}
A_{1}+E_{1} \cos (\omega \tau)+E_{2} \sin (\omega \tau)=0 \\
A_{2}+E_{2} \cos (\omega \tau)-E_{1} \sin (\omega \tau)=0
\end{array}\right.
$$

By calculation, one obtains

$$
\left\{\begin{array}{l}
\sin (\omega \tau)=\frac{E_{1} A_{2}-E_{2} A_{1}}{E_{1}^{2}+E_{2}^{2}}=H_{1}(\omega), \\
\cos (\omega \tau)=-\frac{E_{1} A_{1}+E_{2} A_{2}}{E_{1}^{2}+E_{2}^{2}}=H_{2}(\omega) .
\end{array}\right.
$$

When all parameters are given, by using the fact that $H_{1}(\omega)^{2}+H_{2}(\omega)^{2}=1$, the value of $\omega$ can be easily calculated. Without loss of generality, we assume that $\omega_{i}(i=1,2, \ldots, m)$ are all positive roots of the equation $H_{1}(\omega)^{2}+H_{2}(\omega)^{2}=1$. Note that the value $\tau_{i}$ calculated by the two equations of Eq. (4.23), respectively, should be equal for each $\omega_{i}$. Considering the fact that the codomain of $\arcsin (x)$ and $\arccos (x)$ are $[-\pi / 2, \pi / 2]$ and $[0, \pi]$, respectively, we need to consider the following four cases to find $\tau_{i}$ for each $\omega_{i}$.

Case (i) $H_{1}\left(\omega_{i}\right)>0$ and $H_{2}\left(\omega_{i}\right)>0$. In this case, $\omega_{i} \tau \in(0, \pi / 2)$. One has

$$
\tau_{i}^{(l)}=\frac{\arcsin \left(H_{1}\left(\omega_{i}\right)\right)+2 l \pi}{\omega_{i}}=\frac{\arccos \left(H_{2}\left(\omega_{i}\right)\right)+2 l \pi}{\omega_{i}}, \quad l=0,1,2, \ldots .
$$

Case (ii) $H_{1}\left(\omega_{i}\right)>0$ and $H_{2}\left(\omega_{i}\right)<0$. In this case, $\omega_{i} \tau \in(\pi / 2, \pi)$. One has

$$
\tau_{i}^{(l)}=\frac{\pi-\arcsin \left(H_{1}\left(\omega_{i}\right)\right)+2 l \pi}{\omega_{i}}=\frac{\arccos \left(H_{2}\left(\omega_{i}\right)\right)+2 l \pi}{\omega_{i}}, \quad l=0,1,2, \ldots
$$


Case (iii) $H_{1}\left(\omega_{i}\right)<0$ and $H_{2}\left(\omega_{i}\right)>0$. In this case, $\omega_{i} \tau \in(3 \pi / 2,2 \pi)$. One has

$$
\tau_{i}^{(l)}=\frac{2 \pi+\arcsin \left(H_{1}\left(\omega_{i}\right)\right)+2 l \pi}{\omega_{i}}=\frac{2 \pi-\arccos \left(H_{2}\left(\omega_{i}\right)\right)+2 l \pi}{\omega_{i}}, \quad l=0,1,2, \ldots
$$

Case (iv) $H_{1}\left(\omega_{i}\right)<0$ and $H_{2}\left(\omega_{i}\right)<0$. In this case, $\omega_{i} \tau \in(\pi, 3 \pi / 2)$. One has

$$
\tau_{i}^{(l)}=\frac{\pi-\arcsin \left(H_{1}\left(\omega_{i}\right)\right)+2 l \pi}{\omega_{i}}=\frac{2 \pi-\arccos \left(H_{2}\left(\omega_{i}\right)\right)+2 l \pi}{\omega_{i}}, \quad l=0,1,2, \ldots
$$

Our only interest is the first bifurcation point $\tau_{0}>0$ in this paper. The bifurcation point is defined as

$$
\tau_{0}=\min \left\{\tau_{i}^{(l)}\right\}, \quad i=1, \ldots, m ; l=0,1, \ldots ;
$$

and taking $\omega_{0}=\omega_{i}$, where $\omega_{i}$ corresponds to $\min \left\{\tau_{i}^{(l)}\right\}$.

Based on the above analysis, we have the following lemma.

Lemma 3 If the basic reproduction number $R_{0}>3$, then there exists a value $\tau_{0}>0$, such that the characteristic equation (4.15) at coexistence equilibrium $\varepsilon_{2}=\left(\frac{k}{R_{0}}, \frac{r e k}{d R_{0}^{2}}\left(R_{0}-1\right)\right)$ has at least a pair of pure imaginary roots.

From the previous discussion, to obtain the main results, the following two hypotheses are imposed:

$\left(H_{1}\right) R_{0}>3$.

$\left.\left(H_{2}\right) \operatorname{Re}\left[\frac{d s}{d \tau}\right]\right|_{\left(\tau=\tau_{0}, \omega=\omega_{0}\right)}>0$.

Taking the derivative of $s$ with respect to $\tau$ in Eq. (4.20), one gets

$$
\frac{d s}{d \tau}=\frac{X(s)}{Y(s)}
$$

where

$$
\begin{aligned}
& X(s)=s\left(C_{2} s^{q}+D_{1}\right) e^{-s \tau}, \\
& Y(s)=2 q s^{2 q-1}+q C_{1} s^{q-1}+\left[q C_{2} s^{q-1}-\tau\left(C_{2} s^{q}+D_{1}\right)\right] e^{-s \tau} .
\end{aligned}
$$

Let $X_{1}, X_{2}, Y_{1}, Y_{2}$ be the real and imaginary parts of $X\left(\omega_{0} i\right), Y\left(\omega_{0} i\right)$, respectively. Then, by calculation, one can acquire

$$
\left.\operatorname{Re}\left[\frac{d s}{d \tau}\right]\right|_{\left(\tau=\tau_{0}, \omega=\omega_{0}\right)}=\frac{X_{1} Y_{1}+X_{2} Y_{2}}{Y_{1}^{2}+Y_{2}^{2}}
$$

In summary, we can get the following theorem.

Theorem 2 Suppose $\left(H_{1}\right)-\left(H_{2}\right)$ hold. As $\tau$ increases from zero, there exists a value $\tau_{0}>$ 0 such that system (2.2) exhibits a Hopf bifurcation at coexistence equilibrium $\varepsilon_{2}=$ $\left(\frac{k}{R_{0}}, \frac{r e k}{d R_{0}^{2}}\left(R_{0}-1\right)\right)$ when $\tau=\tau_{0}$. 


\subsection{Bifurcation control for the proposed system}

In this subsection, we introduce a periodic pulse feedback controller $\delta(x(t)-x(t-\tau)) P_{T, p}(t)$ into the first equation of an uncontrolled system (2.2):

$$
\left\{\begin{array}{l}
c \mathcal{D}_{t}^{q} x(t)=r x(t)\left(1-\frac{x(t)}{k}\right)-c(1-m) x(t) y(t-\tau)+\delta(x(t)-x(t-\tau)) P_{T, p}(t), \\
t_{t_{0}} \mathcal{D}_{t}^{q} y(t)=e c(1-m) x(t) y(t-\tau)-d y(t)
\end{array}\right.
$$

where $P_{T, p}(t)$ is defined as $(2.3), \delta<0$.

In order to choose an optimal control strategy, it is necessary to evaluate the control strength of the periodic pulse controller $\delta(x(t)-x(t-\tau)) P_{T, p}(t)$ according to the control effects.

If for some $\delta<0$, the controlled system (4.24) converges to the positive equilibrium $\left(x^{*}, y^{*}\right)$, then, for any $\epsilon>0$, there exists $t_{\epsilon}>0$ such that

$$
|x(t)-x(t-\tau)| \leq \epsilon, \quad t \geq t_{\epsilon}
$$

In this case, the average control strength of the controller $\delta(x(t)-x(t-\tau)) P_{T, p}(t)(\delta<0)$ on each time interval $[n T, n T+T)\left(n \geq \frac{t_{\epsilon}}{T}\right)$ can be measured as follows:

$$
-\frac{\delta}{p} \int_{n T}^{n T+p}|x(t)-x(t-\tau)| d t \leq-\delta \epsilon .
$$

Thus, the average control strength of the controller $\delta(x(t)-x(t-\tau)) P_{T, p}(t)(\delta<0)$ is of $O(\epsilon)$ for different pulse width $p$. This suggests that the control effect may be independent of the pulse width $p$. This claim will be later verified by numerical simulations.

In order to choose an optimal control gain $L_{p}:=\frac{\delta T}{p}$, it is necessary to study the stability of the controlled system (4.24). Since the stability theory is not well-developed for nonlinear fractional-order non-autonomous systems with delays, we need to investigate the linearized system of (4.24) at coexistence equilibrium

$$
\varepsilon_{2}=\left(x^{*}, y^{*}\right)=\left(\frac{k}{R_{0}}, \frac{r e k}{d R_{0}^{2}}\left(R_{0}-1\right)\right) \quad\left(R_{0}>1\right)
$$

which is defined as

$$
\left\{\begin{array}{l}
{ }_{t_{0}}^{c} \mathcal{D}_{t}^{q} x(t)=-a_{1}\left(x(t)-x^{*}\right)-a_{2}\left(y(t-\tau)-y^{*}\right)+\delta(x(t)-x(t-\tau)) P_{T, p}(t), \\
{ }_{t_{0}}^{c} \mathcal{D}_{t}^{q} y(t)=-a_{3}\left(x(t)-x^{*}\right)-a_{4}\left(y(t-\tau)-y^{*}\right)-a_{5}\left(y(t)-y^{*}\right),
\end{array}\right.
$$

where $a_{1}, a_{2}, a_{3}, a_{4}, a_{5}$ are defined as Eq. (4.13).

Noticing that the linearized system (4.25) of the controlled system (4.24) is still a periodic impulse control system, we also need to derive an autonomous linear approximation system of (4.25) so as to study Hopf bifurcation. For this purpose, we further consider an average approximation of the linearized system (4.25).

Set $L:=\frac{1}{T} \int_{0}^{T} \delta P_{T, p}(t) d t=\delta$, which can be regarded as the average control gain of the controller $\delta(x(t)-x(t-\tau)) P_{T, p}(t)(\delta<0)$ on each time interval $[n T, n T+T)(n \geq 0)$. Then, by taking the average of the control gain in the linearized system (4.25), one can derive the 
following autonomous linear system:

$$
\left\{\begin{array}{l}
{ }_{t_{0}}^{c} \mathcal{D}_{t}^{q} \bar{x}(t)=-a_{1}\left(\bar{x}(t)-x^{*}\right)-a_{2}\left(\bar{y}(t-\tau)-y^{*}\right)+L(\bar{x}(t)-\bar{x}(t-\tau)), \\
{ }_{t_{0}}^{c} \mathcal{D}_{t}^{q} \bar{y}(t)=-a_{3}\left(\bar{x}(t)-x^{*}\right)-a_{4}\left(\bar{y}(t-\tau)-y^{*}\right)-a_{5}\left(\bar{y}(t)-y^{*}\right)
\end{array}\right.
$$

which is the average approximation of system (4.25). Therefore, (4.26) can be regarded as the linearized averaging system of the controlled system (4.24).

Next we prove the equivalence of stability of (4.25) and (4.26) in the sense that

$$
\lim _{t \rightarrow+\infty}\left|\bar{x}(t)-x^{*}\right|=0, \quad \lim _{t \rightarrow+\infty}\left|\bar{y}(t)-y^{*}\right|=0
$$

is equivalent to

$$
\lim _{t \rightarrow+\infty}\left|x(t)-x^{*}\right|=0, \quad \lim _{t \rightarrow+\infty}\left|y(t)-y^{*}\right|=0,
$$

where the initial values are taken as $x(t)=\bar{x}(t)=\rho(t)>0$ and $y(t)=\bar{y}(t)=\phi(t)>0(t \in$ $[-\tau, 0])$.

Set $e_{1}(t)=x(t)-\bar{x}(t), e_{2}(t)=y(t)-\bar{y}(t)$. By (4.25) and (4.26), we obtain the error system

$$
\left\{\begin{aligned}
{ }_{t_{0}}^{c} \mathcal{D}_{t}^{q} e_{1}(t)= & -a_{1} e_{1}(t)-a_{2} e_{2}(t-\tau)+\delta P_{T, p}(t)\left(e_{1}(t)-e_{1}(t-\tau)\right) \\
& +\delta\left(P_{T, p}(t)-1\right)(\bar{x}(t)-\bar{x}(t-\tau)) \\
{ }_{t_{0}}^{c} \mathcal{D}_{t}^{q} e_{2}(t)= & -a_{3} e_{1}(t)-a_{4} e_{2}(t-\tau)-a_{5} e_{2}(t)
\end{aligned}\right.
$$

and

$$
\left\{\begin{aligned}
{ }_{t_{0}}^{c} \mathcal{D}_{t}^{q} e_{1}(t)= & -a_{1} e_{1}(t)-a_{2} e_{2}(t-\tau)+\delta\left(P_{T, p}(t)-1\right)(x(t)-x(t-\tau)) \\
& +\delta\left(e_{1}(t)-e_{1}(t-\tau)\right), \\
{ }_{t_{0}}^{c} \mathcal{D}_{t}^{q} e_{2}(t)= & -a_{3} e_{1}(t)-a_{4} e_{2}(t-\tau)-a_{5} e_{2}(t) .
\end{aligned}\right.
$$

Therefore, in order to prove the equivalence of stability of (4.25) and (4.26), it suffices to prove that

$$
\lim _{t \rightarrow+\infty} e_{1}(t)=0, \quad \lim _{t \rightarrow+\infty} e_{2}(t)=0,
$$

where the initial values are taken as $e_{1}(t)=0$ and $e_{2}(t)=0(t \in[-\tau, 0])$.

There are two cases to consider.

Claim A If the solutions $\bar{x}(t)$ and $\bar{y}(t)$ of system (4.26) satisfy

$$
\lim _{t \rightarrow+\infty}\left|\bar{x}(t)-x^{*}\right|=0, \quad \lim _{t \rightarrow+\infty}\left|\bar{y}(t)-y^{*}\right|=0,
$$

then the solutions $e_{1}(t)$ and $e_{2}(t)$ of the error system (4.27) satisfy

$$
\lim _{t \rightarrow+\infty} e_{1}(t)=0, \quad \lim _{t \rightarrow+\infty} e_{2}(t)=0 .
$$


By an argument similar to that used in theoretical analysis of [54], taking the Laplace transform [53] on both sides of system (4.27) gives

$$
\left\{\begin{aligned}
s^{q} E_{1}(s)= & -a_{1} E_{1}(s)-a_{2} e^{-s \tau} E_{2}(s)+\mathcal{L}\left(\delta P_{T, p}(t)\left(e_{1}(t)-e_{1}(t-\tau)\right)\right) \\
& +\mathcal{L}\left(\delta\left(P_{T, p}(t)-1\right)(\bar{x}(t)-\bar{x}(t-\tau))\right) \\
s^{q} E_{2}(s)= & -a_{3} E_{1}(s)-a_{4} e^{-s \tau} E_{2}(s)-a_{5} E_{2}(s)
\end{aligned}\right.
$$

where $E_{1}(s), E_{2}(s)$ are the Laplace transform of $e_{1}(t), e_{2}(t)$ with $E_{1}(s)=\mathcal{L}\left(e_{1}(t)\right), E_{2}(s)=$ $\mathcal{L}\left(e_{2}(t)\right)$.

By (4.29), one gets

$$
E_{1}(s)=-\frac{\left(s^{q}+a_{4} e^{-s \tau}+a_{5}\right) E_{2}(s)}{a_{3}}
$$

and

$$
\begin{aligned}
E_{2}(s)= & \frac{-s^{q} E_{1}(s)-a_{1} E_{1}(s)+\mathcal{L}\left(\delta P_{T, p}(t)\left(e_{1}(t)-e_{1}(t-\tau)\right)\right)}{a_{2} e^{-s \tau}} \\
& +\frac{\mathcal{L}\left(\delta\left(P_{T, p}(t)-1\right)(\bar{x}(t)-\bar{x}(t-\tau))\right)}{a_{2} e^{-s \tau}} .
\end{aligned}
$$

Following the prior assumptions made in theoretical analysis of [54], we make the following prior assumptions: $E_{1}(s), E_{2}(s)$ are bounded. Then, by the final-value theorem of the Laplace transformation [53], it follows from (4.30), (4.31) and (4.13) that

$$
\lim _{t \rightarrow+\infty} e_{1}(t)=\lim _{s \rightarrow 0} s E_{1}(s)=-\frac{\left(a_{4}+a_{5}\right) \lim _{s \rightarrow 0} s E_{2}(s)}{a_{3}}=0,
$$

and hence

$$
\begin{aligned}
\lim _{t \rightarrow+\infty} e_{2}(t)=\lim _{s \rightarrow 0} s E_{2}(s)= & \frac{\lim _{t \rightarrow+\infty}\left(\delta P_{T, p}(t)\left(e_{1}(t)-e_{1}(t-\tau)\right)\right)}{a_{2}} \\
& +\frac{\lim _{t \rightarrow+\infty}\left(\delta\left(P_{T, p}(t)-1\right)(\bar{x}(t)-\bar{x}(t-\tau))\right)}{a_{2}}=0 .
\end{aligned}
$$

Similarly, by taking the Laplace transform [53] on both sides of system (4.28), we can also prove the following.

Claim B If the solutions $x(t)$ and $y(t)$ of system (4.25) satisfy

$$
\lim _{t \rightarrow+\infty}\left|x(t)-x^{*}\right|=0, \quad \lim _{t \rightarrow+\infty}\left|y(t)-y^{*}\right|=0
$$

then the solutions $e_{1}(t)$ and $e_{2}(t)$ of the error system (4.28) satisfy

$$
\lim _{t \rightarrow+\infty} e_{1}(t)=0, \quad \lim _{t \rightarrow+\infty} e_{2}(t)=0 .
$$

Thus, by the equivalence of stability of system (4.25) and system (4.26), we verified the reasonability of the above linearized average approximation from the viewpoint of stability. 
Based on the existence of pure imaginary roots of the characteristic equation for the linearized averaging system (4.26), critical values of the time delay and critical frequencies for the emergence of Hopf bifurcation of system (4.24) can be determined effectively.

Taking the Laplace transform on both sides of system (4.26), the characteristic matrix can be obtained:

$$
\Delta_{3}(s)=\left(\begin{array}{cc}
s^{q}+a_{1}-L+L e^{-s \tau} & a_{2} e^{-s \tau} \\
a_{3} & s^{q}+a_{4} e^{-s \tau}+a_{5}
\end{array}\right) .
$$

Then the characteristic equation of system (4.26) is given by

$$
Q_{1}(s)+Q_{2}(s) e^{-s \tau}+Q_{3}(s) e^{-2 s \tau}=0
$$

where

$$
\begin{aligned}
& Q_{1}(s)=s^{2 q}+\left(a_{1}+a_{5}-L\right) s^{q}+a_{5}\left(a_{1}-L\right), \\
& Q_{2}(s)=\left(a_{4}+L\right) s^{q}+\left(a_{5}-a_{4}\right) L+a_{1} a_{4}-a_{2} a_{3}, \\
& Q_{3}(s)=a_{4} L .
\end{aligned}
$$

By multiplying $e^{s \tau}$ on both sides of Eq. (4.35), we have

$$
Q_{1}(s) e^{s \tau}+Q_{2}(s)+Q_{3}(s) e^{-s \tau}=0 .
$$

Lemma 4 Assume that $\tau>0$. Then the following conclusions hold:

(i) For $1<R_{0}<3$, the characteristic equation (4.35) at coexistence equilibrium $\varepsilon_{2}=\left(\frac{k}{R_{0}}, \frac{r e k}{d R_{0}^{2}}\left(R_{0}-1\right)\right)$ has at least a pair of pure imaginary roots if $L \in\left(\frac{r\left(3-R_{0}\right)}{2 R_{0}},+\infty\right)$.

(ii) For $R_{0}>3$, the characteristic equation (4.35) at coexistence equilibrium $\varepsilon_{2}=\left(\frac{k}{R_{0}}, \frac{r e k}{d R_{0}^{2}}\left(R_{0}-1\right)\right)$ has at least a pair of pure imaginary roots if $L \in\left(\frac{r\left(3-R_{0}\right)}{4 R_{0}},+\infty\right)$.

Proof It is assumed that $s=\omega i=\omega\left(\cos \left(\frac{\pi}{2}\right)+i \sin \left(\frac{\pi}{2}\right)\right)(\omega>0)$ is a root of Eq. (4.36). Thus, Eq. (4.36) gives

$$
\begin{aligned}
\left(a_{4}+L\right) w^{q}\left(\cos \left(\frac{q \pi}{2}\right)+i \sin \left(\frac{q \pi}{2}\right)\right)+\left(a_{5}-a_{4}\right) L+a_{1} a_{4}-a_{2} a_{3} \\
=-\left[w^{2 q}(\cos (q \pi)+i \sin (q \pi))+\left(a_{1}+a_{5}-L\right) w^{q}\left(\cos \left(\frac{q \pi}{2}\right)+i \sin \left(\frac{q \pi}{2}\right)\right)\right. \\
\left.+a_{5}\left(a_{1}-L\right)\right](\cos (\omega \tau)+i \sin (\omega \tau))-a_{4} L(\cos (\omega \tau)-i \sin (\omega \tau)) .
\end{aligned}
$$


Separating real and imaginary parts of Eq. (4.37) gives

$$
\begin{aligned}
\left(a_{4}+L\right) w^{q} \cos \left(\frac{q \pi}{2}\right)+\left(a_{5}-a_{4}\right) L+a_{1} a_{4}-a_{2} a_{3} \\
=-w^{2 q} \cos (q \pi+\omega \tau)-\left(a_{1}+a_{5}-L\right) w^{q} \cos \left(\frac{q \pi}{2}+\omega \tau\right) \\
\left.\quad-a_{5}\left(a_{1}-L\right) \cos (\omega \tau)-a_{4} L\right] \cos (\omega \tau), \\
\left(a_{4}+L\right) w^{q} \sin \left(\frac{q \pi}{2}\right) \\
=-w^{2 q} \sin (q \pi+\omega \tau)-\left(a_{1}+a_{5}-L\right) w^{q} \sin \left(\frac{q \pi}{2}+\omega \tau\right) \\
\quad-a_{5}\left(a_{1}-L\right) \sin (\omega \tau)+a_{4} L \sin (\omega \tau) .
\end{aligned}
$$

By squaring and adding both sides of (4.38), we have

$$
\begin{aligned}
& w^{4 q}+2\left(a_{1}+a_{5}-L\right) \cos \left(\frac{q \pi}{2}\right) w^{3 q}+\left[\left(a_{1}+a_{5}-L\right)^{2}-\left(a_{4}+L\right)^{2}\right. \\
& \left.+2 a_{5}\left(a_{1}-L\right) \cos (q \pi)+2 a_{4} L \cos (q \pi+2 \omega \tau)\right] w^{2 q}+\left[2 a_{5}\left(a_{1}-L\right)\right. \\
& \cdot\left(a_{1}+a_{5}-L\right) \cos \left(\frac{q \pi}{2}\right)+2 a_{4} L\left(a_{1}+a_{5}-L\right) \cos \left(\frac{q \pi}{2}+2 \omega \tau\right) \\
& \left.-2\left(a_{4}+L\right)\left(\left(a_{5}-a_{4}\right) L+a_{1} a_{4}-a_{2} a_{3}\right) \cos \left(\frac{q \pi}{2}\right)\right] w^{q}+a_{5}^{2}\left(a_{1}-L\right)^{2} \\
& +a_{4}^{2} L^{2}+2 a_{5}\left(a_{1}-L\right) a_{4} L \cos (2 \omega \tau)-\left(\left(a_{5}-a_{4}\right) L+a_{1} a_{4}-a_{2} a_{3}\right)^{2}=0 .
\end{aligned}
$$

It suffices to prove that the constant term of Eq. (4.39) is negative, i.e.

$$
\begin{gathered}
a_{5}^{2}\left(a_{1}-L\right)^{2}+a_{4}^{2} L^{2}+2 a_{5}\left(a_{1}-L\right) a_{4} L \cos (2 \omega \tau) \\
-\left(\left(a_{5}-a_{4}\right) L+a_{1} a_{4}-a_{2} a_{3}\right)^{2}<0 .
\end{gathered}
$$

Substituting $a_{i}(i=1,2,3,4,5)$ into Eq. (4.40) and simplifying gives

$$
\left(\frac{r}{R_{0}}-L\right)^{2}+L^{2}-2\left(\frac{r}{R_{0}}-L\right) L \cos (2 \omega \tau)-\left(2 L+\frac{r}{R_{0}}\left(R_{0}-2\right)\right)^{2}<0
$$

There are two cases to consider.

Case (i) If $L \leq 0$ or $L>\frac{r}{R_{0}}$, then we have

$$
\begin{aligned}
& \left(\frac{r}{R_{0}}-L\right)^{2}+L^{2}-2\left(\frac{r}{R_{0}}-L\right) L \cos (2 \omega \tau)-\left(2 L+\frac{r}{R_{0}}\left(R_{0}-2\right)\right)^{2} \\
& \quad \leq\left(\left(\frac{r}{R_{0}}-L\right)-L\right)^{2}-\left(2 L+\frac{r}{R_{0}}\left(R_{0}-2\right)\right)^{2} \\
& \quad=\frac{r}{R_{0}}\left(R_{0}-1\right) \cdot\left(\frac{r}{R_{0}}\left(3-R_{0}\right)-4 L\right) .
\end{aligned}
$$


From this result, we make the inequality (4.41) hold, as long as

$$
\left(R_{0}-1\right) \cdot\left(\frac{r}{R_{0}}\left(3-R_{0}\right)-4 L\right)<0 .
$$

Therefore, for $1<R_{0}<3$, when $L>\max \left\{\frac{r}{R_{0}}, \frac{r}{4 R_{0}}\left(3-R_{0}\right)\right\}=\frac{r}{R_{0}}$, Eq. (4.42) holds; and when $L \leq 0$, Eq. (4.42) does not hold. For $R_{0}>3$, when $\frac{r}{4 R_{0}}\left(3-R_{0}\right)<L \leq 0$ or $L>\frac{r}{R_{0}}$, Eq. (4.42) holds.

Case (ii) If $0<L \leq \frac{r}{R_{0}}$, then we have

$$
\begin{aligned}
& \left(\frac{r}{R_{0}}-L\right)^{2}+L^{2}-2\left(\frac{r}{R_{0}}-L\right) L \cos (2 \omega \tau)-\left(2 L+\frac{r}{R_{0}}\left(R_{0}-2\right)\right)^{2} \\
& \quad \leq\left(\left(\frac{r}{R_{0}}-L\right)+L\right)^{2}-\left(2 L+\frac{r}{R_{0}}\left(R_{0}-2\right)\right)^{2} \\
& \quad=\left(2 L+\frac{r}{R_{0}}\left(R_{0}-1\right)\right) \cdot\left(\frac{r}{R_{0}}\left(3-R_{0}\right)-2 L\right) .
\end{aligned}
$$

From this result, we make the inequality (4.41) hold, as long as

$$
\left(2 L+\frac{r}{R_{0}}\left(R_{0}-1\right)\right) \cdot\left(\frac{r}{R_{0}}\left(3-R_{0}\right)-2 L\right)<0 .
$$

Therefore, for $1<R_{0}<3$, when $\frac{r}{2 R_{0}}\left(3-R_{0}\right)<L \leq \frac{r}{R_{0}}$, Eq. (4.43) holds. For $R_{0}>3$, when $0<L \leq \frac{r}{R_{0}}$, Eq. (4.43) holds.

In summary, for $1<R_{0}<3$, the characteristic equation (4.35) has at least a pair of pure imaginary roots if $L \in\left(\frac{r\left(3-R_{0}\right)}{2 R_{0}}, \frac{r}{R_{0}}\right] \cup\left(\frac{r}{R_{0}},+\infty\right)=\left(\frac{r\left(3-R_{0}\right)}{2 R_{0}},+\infty\right)$; and for $R_{0}>3$, the characteristic equation (4.35) has at least a pair of pure imaginary roots if $L \in\left(\frac{r\left(3-R_{0}\right)}{4 R_{0}}, 0\right] \cup\left(0, \frac{r}{R_{0}}\right] \cup$ $\left(\frac{r}{R_{0}},+\infty\right)=\left(\frac{r\left(3-R_{0}\right)}{4 R_{0}},+\infty\right)$. The proof is complete.

It is assumed that $s=\omega i=\omega\left(\cos \left(\frac{\pi}{2}\right)+i \sin \left(\frac{\pi}{2}\right)\right)(\omega>0)$ is a root of Eq. (4.35). Let $\alpha_{i}, \beta_{i}$ be the real and imaginary parts of $Q_{i}(s)(i=1,2,3)$, respectively, one can obtain

$$
\begin{aligned}
& \alpha_{1}=\omega^{2 q} \cos (q \pi)+\omega^{q}\left(a_{1}+a_{5}-L\right) \cos \left(\frac{q \pi}{2}\right)+a_{5}\left(a_{1}-L\right), \\
& \beta_{1}=\omega^{2 q} \sin (q \pi)+\omega^{q}\left(a_{1}+a_{5}-L\right) \sin \left(\frac{q \pi}{2}\right), \\
& \alpha_{2}=\omega^{q}\left(a_{4}+L\right) \cos \left(\frac{q \pi}{2}\right)+\left(a_{5}-a_{4}\right) L+a_{1} a_{4}-a_{2} a_{3}, \\
& \beta_{2}=\omega^{q}\left(a_{4}+L\right) \sin \left(\frac{q \pi}{2}\right), \\
& \alpha_{3}=a_{4} L, \quad \beta_{3}=0 .
\end{aligned}
$$

It is evident that $s=\omega i=\omega\left(\cos \left(\frac{\pi}{2}\right)+i \sin \left(\frac{\pi}{2}\right)\right)(\omega>0)$ is a root of Eq. (4.36). By substituting $s=\omega i$ into Eq. (4.36) and separating the real and imaginary parts of Eq. (4.36), one has

$$
\left\{\begin{array}{l}
\left(\alpha_{1}+\alpha_{3}\right) \cos (\omega \tau)-\beta_{1} \sin (\omega \tau)+\alpha_{2}=0, \\
\beta_{1} \cos (\omega \tau)+\left(\alpha_{1}-\alpha_{3}\right) \sin (\omega \tau)+\beta_{2}=0 .
\end{array}\right.
$$


It follows from Eq. (4.44) that

$$
\left\{\begin{array}{l}
\sin (\omega \tau)=-\frac{\beta_{2}\left(\alpha_{1}+\alpha_{3}\right)-\beta_{1} \alpha_{2}}{\alpha_{1}^{2}+\beta_{1}^{2}-\alpha_{3}^{2}}=H_{1}(\omega), \\
\cos (\omega \tau)=-\frac{\alpha_{2}\left(\alpha_{1}-\alpha_{3}\right)+\beta_{1} \beta_{2}}{\alpha_{1}^{2}+\beta_{1}^{2}-\alpha_{3}^{2}}=H_{2}(\omega) .
\end{array}\right.
$$

By Eq. (4.45), the first bifurcation point $\tau_{0}^{*}$ and the corresponding critical frequency $\omega_{0}^{*}$ can be obtained by the method used in Sect. 4.2.

From the previous discussion, to obtain the main results, the following hypotheses are imposed:

$\left(H_{3}\right) L \in\left(\frac{r\left(3-R_{0}\right)}{2 R_{0}},+\infty\right)$ for $1<R_{0}<3$, or $L \in\left(\frac{r\left(3-R_{0}\right)}{4 R_{0}},+\infty\right)$ for $R_{0}>3$;

$\left.\left(H_{4}\right) \operatorname{Re}\left[\frac{d s}{d \tau}\right]\right|_{\left(\tau=\tau_{0}^{*}, \omega=\omega_{0}^{*}\right)}>0$.

Differentiating Eq. (4.35) with respect to $\tau$, by implicit function theorem, we have

$$
\frac{d s}{d \tau}=\frac{M(s)}{N(s)}
$$

where

$$
\begin{aligned}
& M(s)=s\left(Q_{2}(s) e^{-s \tau}+2 Q_{3}(s) e^{-2 s \tau}\right), \\
& N(s)=Q_{1}^{\prime}(s)+\left(Q_{2}^{\prime}(s)-\tau Q_{2}(s)\right) e^{-s \tau}+\left(Q_{3}^{\prime}(s)-2 \tau Q_{3}(s)\right) e^{-2 s \tau},
\end{aligned}
$$

and $Q_{i}^{\prime}(s)$ is the derivative of $Q_{i}(s)(i=1,2,3)$.

Set $M_{i}, N_{i}(i=1,2)$ are the real and imaginary parts of $M\left(\omega_{0}^{*} i\right), N\left(\omega_{0}^{*} i\right)$, respectively. Then, by calculation, one can acquire

$$
\left.\operatorname{Re}\left[\frac{d s}{d \tau}\right]\right|_{\left(\tau=\tau_{0}^{*}, \omega=\omega_{0}^{*}\right)}=\frac{M_{1} N_{1}+M_{2} N_{2}}{N_{1}^{2}+N_{2}^{2}}
$$

Based on the above analysis, we can obtain the following theorem.

Theorem 3 Suppose that $\left(H_{3}\right),\left(H_{4}\right)$ hold. As $\tau$ increases from zero, there exists a value $\tau_{0}^{*}>0$ such that system (4.24) exhibits a Hopf bifurcation at coexistence equilibrium $\varepsilon_{2}=$ $\left(\frac{k}{R_{0}}, \frac{r e k}{d R_{0}^{2}}\left(R_{0}-1\right)\right)$ when $\tau=\tau_{0}^{*}$.

\section{Numerical simulations}

In this section, two numerical examples are given to validate our theoretical results. The Adama-Bashforth-Moulton predictor-corrector scheme in [55] is utilized.

Example 1 In this example, we choose time delay $\tau$ as a bifurcation parameter to investigate Hopf bifurcation of system (2.2). The system parameter values are chosen as $r=1.2$, $k=20, c=0.9, d=0.3, e=0.3, m=0.8, q=0.98$. It is easy to get the basic reproduction number $R_{0}=\frac{k e c(1-m)}{d}=3.6>3$ and the coexistence equilibrium $\varepsilon_{2}=\left(\frac{k}{R_{0}}, \frac{r e k}{d R_{0}^{2}}\left(R_{0}-1\right)\right)=$ $(5.5556,4.8148)$. By calculating, we get the critical frequency $\omega_{0}=0.2595$ and the bifurcation point $\tau_{0}=4.9967$. It is easy to verify that conditions of $\left(H_{1}\right),\left(H_{2}\right)$ are satisfied. Therefore, it follows from Theorem 2 that system (2.2) exhibits a Hopf bifurcation at coexistence equilibrium $\varepsilon_{2}=(5.5556,4.8148)$ when $\tau=\tau_{0}=4.9967$, which is displayed in Figs. $1-4$. 

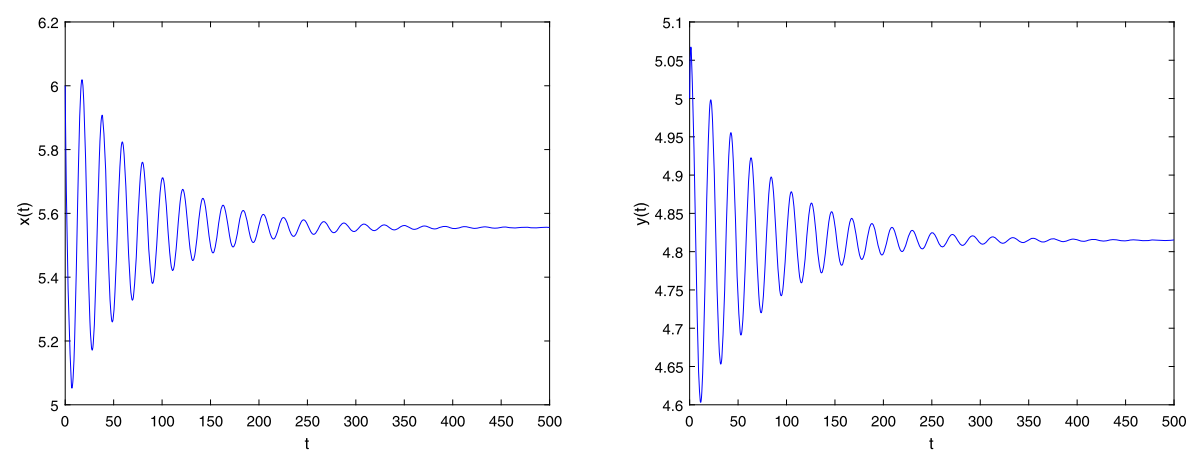

Figure 1 Waveform plots of system $(2.2)$ with initial values $(6.46,5.1)$ in Example 1. The delay is chosen as: $\tau=3.8<\tau_{0}=4.9967$. This figure illustrates that the coexistence equilibrium $\varepsilon_{2}=(5.5556,4.8148)$ is asymptotically stable

Figure 2 Portrait of system (2.2) with initial values $(6.46,5.1)$ in Example 1. The delay is chosen as: $\tau=3.8$ $<\tau_{0}=4.9967$. This figure illustrates that the coexistence equilibrium $\varepsilon_{2}=(5.5556,4.8148)$ is asymptotically stable
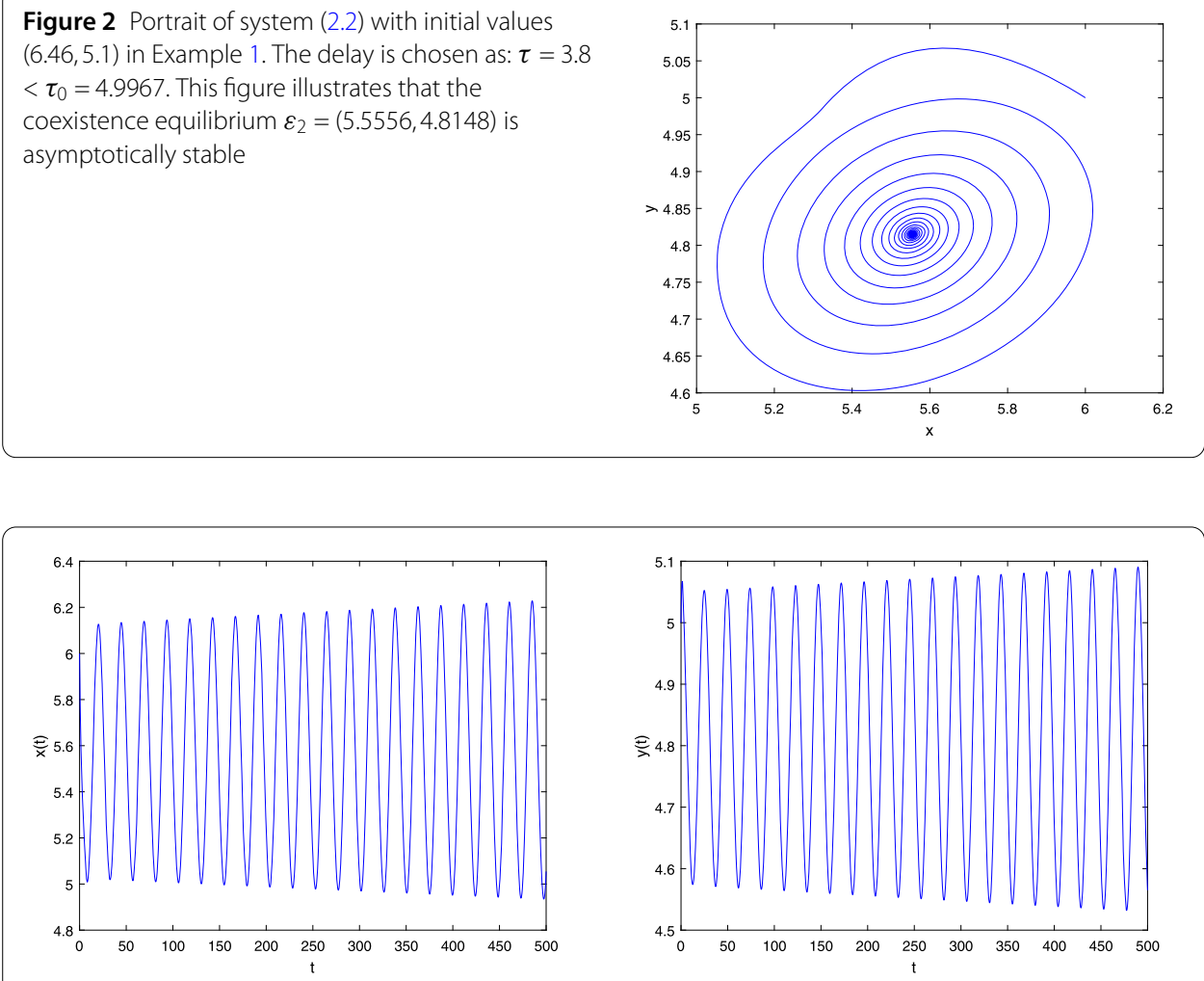

Figure 3 Waveform plots of system $(2.2)$ with initial values $(6.46,5.1)$ in Example 1. The delay is chosen as: $\tau=5.1>\tau_{0}=4.9967$. This figure illustrates that the coexistence equilibrium $\varepsilon_{2}=(5.5556,4.8148)$ is unstable

The biological significance of Hopf bifurcation in this model is that predator and prey may coexist, showing cyclical oscillatory balance behavior.

Example 2 In order to highlight the control effect, all system parameters are the same as in Example 1, that is, $r=1.2, k=20, c=0.9, d=0.3, e=0.3, m=0.8, q=0.98, \tau=5.1>\tau_{0}$ and $T=10$. It is easy to get the basic reproduction number $R_{0}=\frac{k e c(1-m)}{d}=3.6>3$ and the coexistence equilibrium $\varepsilon_{2}=\left(\frac{k}{R_{0}}, \frac{r e k}{d R_{0}^{2}}\left(R_{0}-1\right)\right)=(5.5556,4.8148)$. To achieve desirable 
Figure 4 Portrait of system (2.2) with initial values (6.46, 5.1) in Example 1. The delay is chosen as: $\tau=5.1>\tau_{0}$ $=4.9967$. This figure illustrates that system (2.2) exhibits a Hopf bifurcation

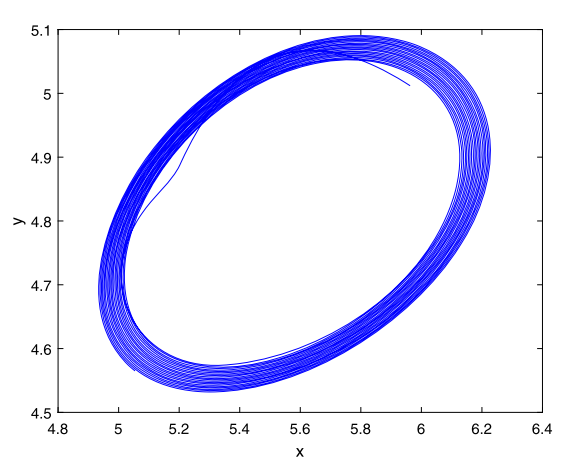

Table 1 The impact of the feedback gain $L$ on the first bifurcation point $\tau_{0}^{*}$ and critical frequency $\omega_{0}^{*}$ for the controlled system (4.24). This table lists the values of the first bifurcation point $\tau_{0}^{*}$ and critical frequency $\omega_{0}^{*}$ corresponding to some feedback gain $L$, which are calculated from the linearized average system (4.26) of the controlled system (4.24) with the parameter values $r=1.2, k=20, c=0.9$, $d=0.3, e=0.3, m=0.8, q=0.98$

\begin{tabular}{lll}
\hline Feedback gain $L$ & Bifurcation point $\tau_{0}^{*}$ & Critical frequency $\omega_{0}^{*}$ \\
\hline-0.045 & 9.470591051 & 0.1737564826 \\
-0.04 & 8.215802361 & 0.1901197022 \\
-0.035 & 7.413432297 & 0.2027989139 \\
-0.03 & 6.830468978 & 0.2134653512 \\
-0.025 & 6.376632347 & 0.2228352248 \\
-0.02 & 6.007515697 & 0.2312894556 \\
-0.015 & 5.698047907 & 0.2390574929 \\
-0.01 & 5.432721606 & 0.2462893820 \\
-0.005 & 5.201297255 & 0.2530891673 \\
0 & 4.996672114 & 0.2595322466 \\
0.1 & 3.049979623 & 0.3549183506 \\
0.2 & 2.293269224 & 0.4267425452 \\
0.3 & 1.856580079 & 0.4895983980 \\
0.4 & 1.564977940 & 0.5471409136 \\
0.5 & 1.354249235 & 0.6009013984 \\
\hline
\end{tabular}

dynamics, we next consider the effect of the feedback gain $L$ on the bifurcation point. It can be seen from Table 1 that the system (2.2) is successfully controlled and the stable domain becomes larger as the feedback gain decreases, and the values of bifurcation point increases as the feedback gain decreases for $L<0$. And the effects of bifurcation control become better as the feedback gain $L$ decreases, which is displayed in Fig. 5. However, for $L>0$, as $L$ increases, the bifurcation point decreases, which implies that the Hopf bifurcation occurs in advance. When $p$ and $T$ are fixed, the feedback gain of the periodic pulse feedback controller at $n T \leq t<n T+p$ is $\frac{\delta T}{p}$. As shown in Figs. 6-8, as $\delta$ decreases, the system converges faster to a steady state.

Remark 1 From an ecological point of view, when the feedback gain $\frac{\delta T}{p}<0$, there are two different control effects. If $x(t)-x(t-\tau)<0$, then $\frac{\delta T}{p}(x(t)-x(t-\tau))>0$, which implies that the control effect is the stocking of prey. If $x(t)-x(t-\tau)>0$, then $\frac{\delta T}{p}(x(t)-x(t-\tau))<0$, which implies that the control effect is the harvesting of prey. As shown in Figs. 5-8, the greater the proportion of stocking or harvesting is, the faster the system converges to a steady state. 

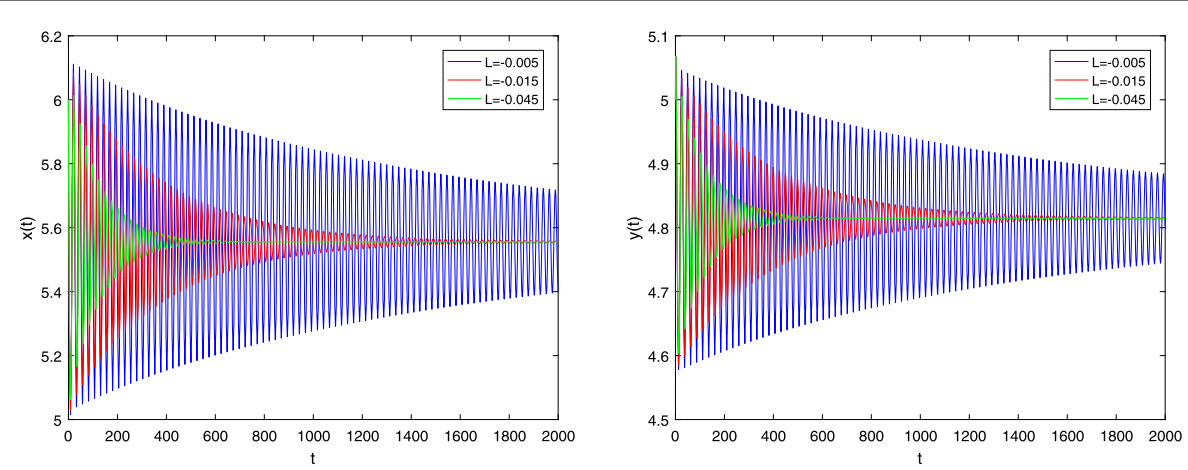

Figure 5 Waveform plots of system $(4.24)$ with initial values $(6.46,5.1)$ in Example 2. This figure illustrates the control effects for the pulse width $p=10$ and three different average control gain $L=-0.005,-0.015,-0.045$
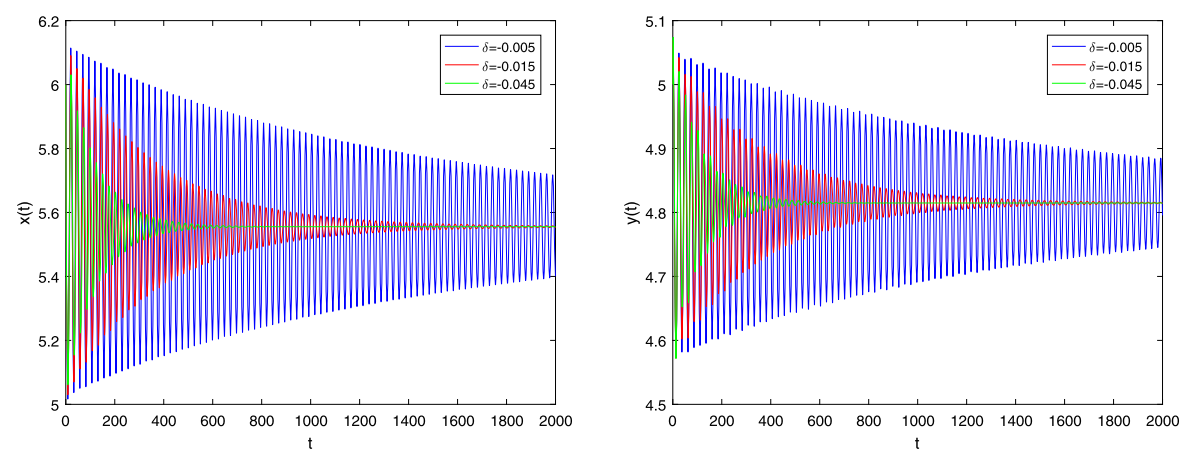

Figure 6 Waveform plots of system $(4.24)$ with initial values $(6.46,5.1)$ in Example 2. This figure illustrates the control effects for the pulse width $p=2$ and three different control gain $\delta=-0.005,-0.015,-0.045$
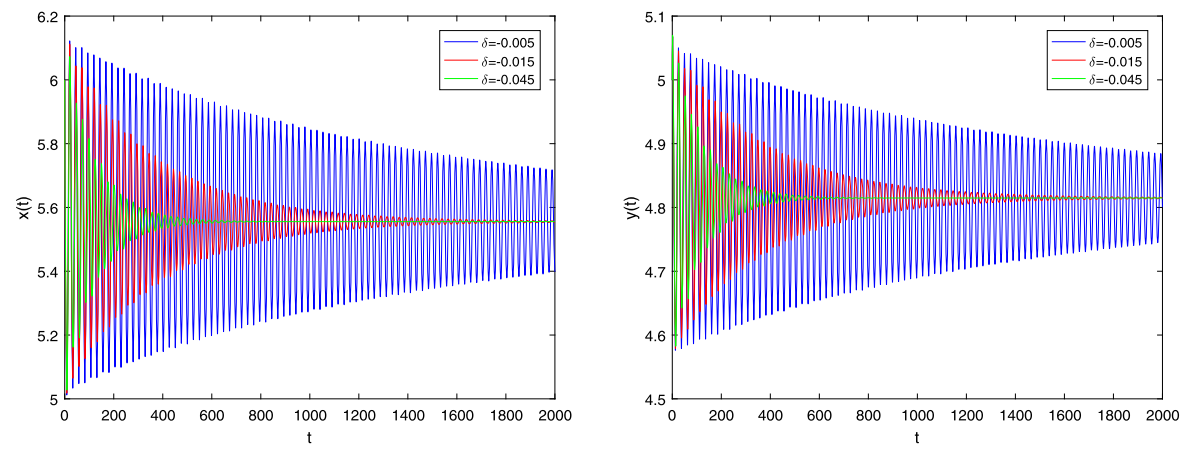

Figure 7 Waveform plots of system $(4.24)$ with initial values $(6.46,5.1)$ in Example 2. This figure illustrates the control effects the pulse width $p=5$ and three different control gain $\delta=-0.005,-0.015,-0.045$

Remark 2 For a fixed period $T$, regardless of the values of the pulse width $p$, the controllers always achieve the same control effect at the same time, as showed in Figs. 5-8. Indeed, when the control time $p$ in each period is decreased, the control force $\left|\frac{\delta T}{p}\right|$ is also increased correspondingly, which can guarantee the same control effect achieved by taking different $p$. 

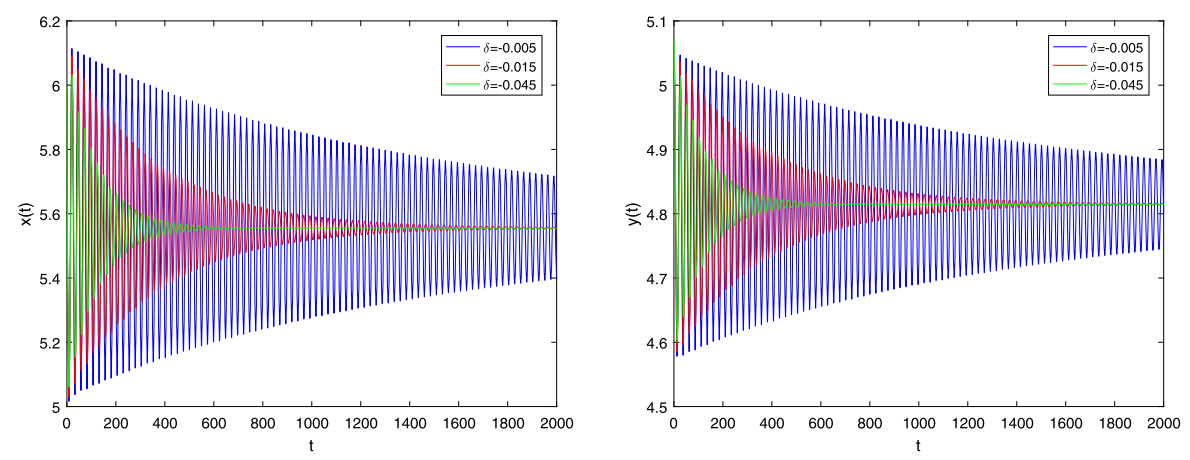

Figure 8 Waveform plots of system $(4.24)$ with initial values $(6.46,5.1)$ in Example 2. This figure illustrates the control effects for the pulse width $p=8$ and three different control gain $\delta=-0.005,-0.015,-0.045$

\section{Conclusions and discussions}

In this paper, sufficient conditions on the existence and uniqueness of the solution, and the emergence of Hopf bifurcation have been established for a fractional-order delay predator-prey model incorporating a prey refuge. The periodic pulse delay feedback control issue of Hopf bifurcations for the model has also been investigated by theoretical analysis and numerical simulation.

This paper mainly studied two aspects: delay-induced Hopf bifurcation and periodic pulse delay feedback control of Hopf bifurcation. Taking the time delay as the bifurcation parameter, Hopf bifurcations for system (2.2) and (4.24) are studied. The explicit conditions for the existence of pure imaginary roots of the characteristic equations for linearized systems are given using the basic reproduction number $R_{0}$. Critical values of the time delay and critical frequencies for the emergence of Hopf bifurcation are determined.

From the perspective of ecological management and species richness, a stable positive equilibrium is needed to maintain the sustainable development of ecosystems. We design a periodic pulse delay feedback controller to drive the predator-prey system from periodic oscillation to steady state by adjusting the harvesting and stocking work of the prey population. Managers can develop policies to encourage or restrain harvesting efforts, such as adjusting market prices, subsidizing harvests, and reducing environmental pollution, in order to maintain a steady state of biological populations. In order to control the onset of the Hopf bifurcation, a periodic pulse delay feedback controller is introduced into uncontrolled system (2.2). It is shown that the Hopf bifurcation can be effectively controlled by selecting appropriate feedback gain and pulse width.

The classic linear delay feedback controller requires real-time control, while our proposed periodic pulse delay feedback controller, due to the characteristic of periodic intermittent control, is more flexible than the linear delay feedback controller. And under the determined period, the periodic pulse delay feedback controller can achieve the same control effect, regardless of the value of the pulse width $p$. To achieve the optimal timesaving effect without reducing control effect, the control time interval in each period can be flexibly selected according to the actual problem.

The relationship between stability of the nonlinear systems (2.2), (4.24) and their linearized systems is not theoretically analyzed. Moreover, we only use numerical simulation to illustrate the conclusion that the control effect of periodic pulse delay feedback controller $\delta(x(t)-x(t-\tau)) P_{T, p}(t)$ to system (2.2) is independent of the pulse width $p$. We 
guess that the conclusion can be proved by studying the stability of the controlled system (4.24). These problems are still open and challenging, since the stability theory is not welldeveloped for nonlinear fractional-order autonomous or non-autonomous systems with delays.

\section{Appendices}

The following Matlab codes are compiling based on the Adama-Bashforth-Moulton predictor-corrector scheme in [55].

\section{A.1 A sample Matlab code for the graphical solutions of system (2.2)}

clear all;

close all;

clc;

TSim $=500 ; \%$ Simulation duration

$\mathrm{h}=0.05 ; \quad \%$ Step size

$\mathrm{N}=\mathrm{fix}(\mathrm{TSim} / \mathrm{h}) ; \quad$ \% Number of iterations

$\mathrm{n}=1: \mathrm{N}+1$;

$\mathrm{t}=(\mathrm{n}-1) * \mathrm{~h} ; \quad$ \% Discrete-time sequence

alpha $=0.98 ; \quad$ \% Fractional order

tau $=5.1 ; \quad \%$ Time delay

$\mathrm{K}=\operatorname{round}(\operatorname{tau} / \mathrm{h})$;

\% Specifying arrays for storing plotting data

$\mathrm{X}=\mathrm{zeros}(1, \mathrm{~N}+1)$;

$\mathrm{Y}=\mathrm{zeros}(1, \mathrm{~N}+1)$;

$\%$ Initial values

$\mathrm{x} 0=6.46$;

$\mathrm{y} 0=5.1$

$\mathrm{X}(1)=\mathrm{x} 0$;

$\mathrm{Y}(1)=\mathrm{y} 0$;

\% Specifying arrays for storing initial values

ivx=zeros $(1, \mathrm{~K})$;

ivy=zeros $(1, \mathrm{~K})$;

for $j=1: K$

$\operatorname{ivx}(j)=x 0$;

$\operatorname{ivy}(j)=y 0$;

end

\% Specifying arrays for storing the state variables $x(t), y(t), x(t-\tau), y(t-\tau)$

$\mathrm{x}=\mathrm{zeros}(1, \mathrm{~N})$;

$\mathrm{y}=\mathrm{zeros}(1, \mathrm{~N})$;

$\mathrm{xd}=\operatorname{zeros}(1, \mathrm{~N})$;

$y d=\operatorname{zeros}(1, N)$;

$\%$ Specifying arrays for storing the right-side function values

$\mathrm{fp}=\operatorname{zeros}(1, \mathrm{~N})$; 
fn1d=zeros $(1, N)$;

$\mathrm{gp}=\operatorname{zeros}(1, \mathrm{~N})$;

gn1d=zeros $(1, \mathrm{~N})$;

$\mathrm{A}=\mathrm{zeros}(1, \mathrm{~N}) ; \quad \%$ An array storing weight coefficients $a_{i, n+1}$ of the corrector term

$\mathrm{B}=\mathrm{zeros}(1, \mathrm{~N}) ; \quad \%$ An array storing weight coefficients $b_{i, n+1}$ of the predictor term

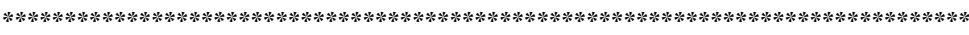

\% Starting the first iteration

a01=alpha; \% The weight coefficient $a_{0,1}$ of the corrector term

b01=halpha/alpha; $\quad \%$ The weight coefficient $b_{0,1}$ of the predictor term

$\%$ Calculate the function values without the predictive values at the first iteration

$\mathrm{f} 10 \mathrm{~d}=\mathrm{f}(\mathrm{x} 0$,ivy $(\mathrm{K}))$;

$\mathrm{g} 10 \mathrm{~d}=\mathrm{g}(\mathrm{x} 0, \mathrm{y} 0, \mathrm{ivy}(\mathrm{K}))$;

$\%$ Calculate the predictive values of $x(1), y(1)$

$\mathrm{xh}(1)=\mathrm{x} 0+1 /$ gamma(alpha)"b01"f10d;

$\mathrm{yh}(1)=y 0+1 /$ gamma(alpha)*b01"g10d;

\% Calculate the function values with the predictive values at the first iteration

$\mathrm{fp} 0 \mathrm{~d}=\mathrm{f}(\mathrm{xh}(1)$,ivy $(\mathrm{K}-1))$;

$\operatorname{gp} 0 \mathrm{~d}=\mathrm{g}(\mathrm{xh}(1), \operatorname{yh}(1), \mathrm{ivy}(\mathrm{K}-1))$;

\% Calculate the values of the state variables $x(t), y(t)$ at the first iteration

$\mathrm{x}(1)=\mathrm{x} 0+\mathrm{h}$ ^alpha/gamma(alpha+2)*fp0d+h^alpha/gamma(alpha+2)*a01*f10d;

$y(1)=y 0+h$ ^alpha/gamma(alpha+2)"gp0d+h^alpha/gamma(alpha+2)*a01*g10d;

$\mathrm{X}(2)=\mathrm{x}(1)$;

$\mathrm{Y}(2)=\mathrm{y}(1)$;

$\%$ End of the first iteration

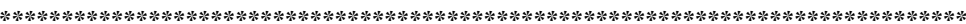

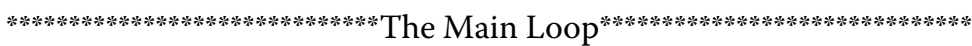

for $\mathrm{n}=1: \mathrm{N}-1$

$\mathrm{A} 0=\mathrm{n}^{\wedge}(\mathrm{alpha}+1)-\left(\mathrm{n}\right.$-alpha)*(n+1)^alpha; $\%$ The weight coefficients $a_{0, n+1}$ of the corrector term

B0=h^alpha/alpha*((n+1)^alpha-n^alpha); \% The weight coefficients $b_{0, n+1}$ of the predictor term

for $\mathrm{i}=1: \mathrm{n}$

$$
\mathrm{A}(\mathrm{i})=(\mathrm{n}-\mathrm{i}+2)^{\wedge}(\text { alpha }+1)+(\mathrm{n}-\mathrm{i})^{\wedge}(\text { alpha }+1)-2^{*}(\mathrm{n}-\mathrm{i}+1)^{\wedge}(\text { alpha }+1) ; \quad \% \text { The weight co- }
$$

efficients $a_{i, n+1}$ of the corrector term

$\mathrm{B}(\mathrm{i})=\mathrm{h}$ alpha* ((n-i+1)^alpha-(n-i)^alpha)/alpha; $\quad \%$ The weight coefficients $b_{i, n+1}$

of the predictor term

end

\% This loop is used for calculating the state variables with delay

for $j=1: n+1$

if $j<K$

$\operatorname{xd}(\mathrm{j})=\mathrm{ivx}(\mathrm{K}-\mathrm{j})$;

$y d(j)=i v y(K-j)$;

elseif $j==K$

$\mathrm{xd}(\mathrm{j})=\mathrm{x} 0$; 


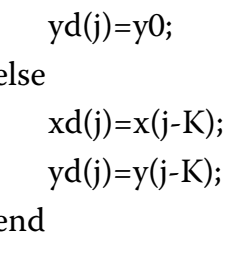

$\%$ Calculate the function values without the predictive values at $(n+1)$ th iteration fn1d $(n)=f(x(n), y d(n))$;

$\operatorname{gn} 1 d(n)=g(x(n), y(n), y d(n))$;

$\%$ Calculate the predictive values of $x(n+1), y(n+1)$

$\mathrm{xh}(\mathrm{n}+1)=\mathrm{x} 0+1 /$ gamma $($ alpha $) *\left(\mathrm{BO} * \mathrm{f} 10 \mathrm{~d}+\mathrm{B}^{*} \mathrm{fn} 1 \mathrm{~d}^{\prime}\right)$;

$y h(n+1)=y 0+1 /$ gamma $($ alpha $) *(B 0 *$ g10d+B"gn1d');

\% Calculate the function values with the predictive values at $(n+1)$ th iteration

$f p(n+1)=f(x h(n+1), y d(n+1))$;

$\mathrm{gp}(\mathrm{n}+1)=\mathrm{g}(\mathrm{xh}(\mathrm{n}+1), \mathrm{yh}(\mathrm{n}+1), \mathrm{yd}(\mathrm{n}+1)) ;$

\% Calculate the values of the state variables $x(t), y(t)$ at $(n+1)$ th iteration

$\mathrm{x}(\mathrm{n}+1)=\mathrm{x} 0+\mathrm{h}$ alpha/gamma $($ alpha +2$) * \mathrm{fp}(\mathrm{n}+1)$

+h^alpha/gamma(alpha +2$) *\left(A 0 * f 10 d+A * f n 1 d^{\prime}\right)$;

$\mathrm{y}(\mathrm{n}+1)=\mathrm{y} 0+\mathrm{h}$ alpha/gamma $($ alpha +2$)$ "gp $(\mathrm{n}+1)$

+ h alpha/gamma(alpha+2)*(A0*g10d+A*gn1d');

$X(n+2)=x(n+1)$;

$Y(n+2)=y(n+1)$;

end

figure(1)

$\operatorname{plot}(t(1: e n d), X(1: e n d), ' b-')$

hold on

xlabel('t'),ylabel('x(t)')

hold off

figure(2)

$\operatorname{plot}\left(\mathrm{t}(1: \mathrm{end}), \mathrm{Y}(1:\right.$ end $\left.), \mathrm{b}^{-} \mathrm{-}^{\prime}\right)$

hold on

xlabel('t'),ylabel('y(t)')

hold off

figure(3)

plot(X(1:end),Y(1:end),'b-',LineWidth'0.5)

hold on

xlabel('x'),ylabel('y')

hold off

\% Define the functions $f(x, y d), g(x, y, y d)$ used in the previous code

function $U=f(x, y d) \%$ The first function on the right side of the model

$\mathrm{r}=1.2 ; \mathrm{k}=20 ; \mathrm{c}=0.9 ; \mathrm{m}=0.8 ; \mathrm{e}=0.3 ; \mathrm{d}=0.3 ; \%$ Parameter values in the model

$\mathrm{U}=\mathrm{r}^{*} \mathrm{x}^{*}(1-\mathrm{x} / \mathrm{k})-\mathrm{c}^{*}(1-\mathrm{m}){ }^{*} \mathrm{x}^{*} \mathrm{yd}$; 
end

function $\mathrm{V}=\mathrm{g}(\mathrm{x}, \mathrm{y}, \mathrm{yd}) \%$ The second function on the right side of the model

$\mathrm{r}=1.2 ; \mathrm{k}=20 ; \mathrm{c}=0.9 ; \mathrm{m}=0.8 ; \mathrm{e}=0.3 ; \mathrm{d}=0.3$;

$\mathrm{V}=\mathrm{e}^{*} \mathrm{c}^{* *}(1-\mathrm{m})^{* *} \mathrm{x}^{*} \mathrm{yd}-\mathrm{d}^{*} \mathrm{y}$

end

\section{A.2 A sample Matlab code for the graphical solutions of system (4.24)}

A sample Matlab code for system (4.24) can be easily obtained by modifying the first function in the previous code for system (2.2):

function $\mathrm{U}=\mathrm{f}(\mathrm{t}, \mathrm{x}, \mathrm{xd}, \mathrm{yd})$

$$
\mathrm{r}=1.2 ; \mathrm{k}=20 ; \mathrm{c}=0.9 ; \mathrm{m}=0.8 ; \mathrm{e}=0.3 ; \mathrm{d}=0.3 ; \text { delta }=-0.005 ; \text { Period }=10 ; \mathrm{p}=8 \text {; }
$$$$
\left.\mathrm{U}=\mathrm{r}^{* *} \mathrm{x}^{*}(1-\mathrm{x} / \mathrm{k})-\mathrm{C}^{* *}(1-\mathrm{m})^{*} \mathrm{x}^{*} \mathrm{yd}+\operatorname{delta}^{*}(\operatorname{Period} / \mathrm{p})^{*}(\mathrm{x}-\mathrm{xd})\right)^{*}((\bmod (\mathrm{t}, \text { Period })>=0
$$

$\& \bmod (\mathrm{t}$, Period $)<\mathrm{p}))$

end

In this case, the parameter value TSim is taken as TSim $=200 *$ Period.

\section{Acknowledgements}

The authors would like to thank the editor and the anonymous reviewers for their valuable comments and constructive suggestions.

Funding

This research is supported by the National Natural Science Foundation of China (Grant Nos. 10971085, 11561034).

Availability of data and materials

Not applicable.

Competing interests

The authors declare that they have no competing interests.

Authors' contributions

All authors contributed equally. All authors read and approved the final manuscript.

\section{Publisher's Note}

Springer Nature remains neutral with regard to jurisdictional claims in published maps and institutional affiliations.

Received: 16 August 2019 Accepted: 7 November 2019 Published online: 19 November 2019

\section{References}

1. Edelman, M.: Fractional maps as maps with power-law memory. Physics 8, 79-120 (2013)

2. Rihan, F.A., Baleanu, D., Lakshmanan, S., Rakkiyappan, R.: On fractional SIRC model with salmonella bacterial infection. Abstr. Appl. Anal. 2014, 136263 (2014)

3. Rihan, F.A., Lakshmanan, S., Hashish, A.H., Rakkiyappan, R., Ahmed, E.: Fractional-order delayed predator-prey systems with Holling type-II functional response. Nonlinear Dyn. 80, 777-789 (2015)

4. Nosrati, K., Shafiee, M.: Dynamic analysis of fractional-order singular Holling type-Il predator-prey system. Appl. Math. Comput. 313, 159-179 (2017)

5. Padisak, J.: Seasonal succession of phytoplankton in a large shallow lake (Balaton, Hungary) —a dynamic approach to ecological memory, its possible role and mechanisms. J. Ind. Ecol. 80, 217-230 (1992)

6. Peterson, G.D.: Contagious disturbance, ecological memory, and the emergence of landscape pattern. Ecosystems 5 , 329-338 (2002)

7. Kilbas, A.A., Srivastava, H.M., Trujillo, J.J.: Theory and Applications of Fractional Differential Equations. Elsevier, Amsterdam (2006)

8. Podlubny, l.: Fractional Differential Equations. Academic Press, New York (1993)

9. Wang, J.L., Li, H.F.: Surpassing the fractional derivative: concept of the memory-dependent derivative. Comput. Math. Appl. 62, 1562-1567 (2011)

10. Sabatier, J., Agrawal, O.P., Tenreiro Machado, J.A.: Advances in fractional calculus: theoretical developments and applications in physics and engineering. SIAM Rev. 50, 613-615 (2008)

11. Yang, X.J., Srivastava, H.M., Tenreiro Machado, J.A.: A new fractional derivative without singular kernel: application to the modelling of the steady heat flow. Therm. Sci. 20(2), 753-756 (2015)

12. Yang, X.J., Gao, F., Tenreiro Machado, J.A., Baleanu, D.: A new fractional derivative involving the normalized sinc function without singular kernel. Eur. Phys. J. Spec. Top. 226(16-18), 3567-3575 (2017) 
13. Yang, X.J., Gao, F., Srivastava, H.M.: A new computational approach for solving nonlinear local fractional PDEs. J. Comput. Appl. Math. 339, 285-296 (2018)

14. Yang, X.J., Gao, F., Ju, Y., Zhou, H.W.: Fundamental solutions of the general fractional-order diffusion equations. Math. Methods Appl. Sci. 41(18), 9312-9320 (2018)

15. Yang, X.J.: Fractional derivatives of constant and variable orders applied to anomalous relaxation models in heat transfer problems. Therm. Sci. 21(3), 1161-1171 (2016)

16. Yang, X.J., Machado, J.A.T.: A new fractional operator of variable order: application in the description of anomalous diffusion. Physica A 481, 276-283 (2017)

17. Panja, P.: Stability and dynamics of a fractional-order three-species predator-prey model. Theory Biosci. 138, 251-259 (2019)

18. Javidi, M., Nyamoradi, N.: Dynamic analysis of a fractional order prey-predator interaction with harvesting. Appl. Math. Model. 37, 8946-8956 (2013)

19. Li, H.L., Zhang, L., Hu, C., Jiang, Y.L., Teng, Z.: Dynamical analysis of a fractional-order predator-prey mode incorporating a prey refuge. J. Appl. Math. Comput. 54, 435-449 (2016)

20. Panja, P.: Dynamics of a fractional order predator-prey model with intraguild predation. Int. J. Model. Simul. 39, 256-268 (2019)

21. Vargas-De-LeÓn, C.: Volterra-type Lyapunov functions for fractional-order epidemic systems. Commun. Nonlinear Sci. Numer. Simul. 24, 75-85 (2015)

22. Huo, J., Zhao, H., Zhu, L.: The effect of vaccines on backward bifurcation in a fractional order HIV model. Nonlinear Anal., Real World Appl. 26, 289-305 (2015)

23. Abdelouahab, M.S., Hamri, N.E., Wang, J.: Hopf bifurcation and chaos in fractional-order modified hybrid optical system. Nonlinear Dyn. 69, 275-284 (2012)

24. Moustafa, M., Mohd, M.H., Ismail, A.l., Abdullah, F.A.: Dynamical analysis of a fractional-order Rosenzweig-MacArthur model incorporating a prey refuge. Chaos Solitons Fractals 109, 1-13 (2018)

25. Choi, S.K., Kang, B., Koo, N.: Stability for Caputo fractional differential systems. Abstr. Appl. Anal. 2014, 631419 (2014)

26. Elsadany, A., Matouk, A.: Dynamical behaviors of fractional-order Lotka-Volterra predator-prey model and its discretization. J. Appl. Math. Comput. 49, 269-283 (2015)

27. Rihan, F.A.: Sensitivity analysis of dynamic systems with time lags. J. Comput. Appl. Math. 151, 445-462 (2003)

28. Rihan, F.A., Anwar, M.N.: Qualitative analysis of delayed SIR epidemic model with a saturated incidence rate. Int. J. Differ. Equ. 2012, 408637 (2012)

29. Zhang, J.F.: Bifurcation analysis of a modified Holling-Tanner predator-prey model with time delay. Appl. Math Model. 36, 1219-1231 (2012)

30. Deng, W., Li, C., Lü, J.: Stability analysis of linear fractional differential system with multiple time delays. Nonlinear Dyn. 48, 409-416 (2006)

31. Xiao, Z., Xie, X., Xue, Y: Stability and bifurcation in a Holling type-II predator-prey model with Allee effect and time delay. Adv. Differ. Equ. 2018, 288 (2018)

32. Wang, L., Feng, G.: Stability and Hopf bifurcation for a ratio-dependent predator-prey system with stage structure and time delay. Adv. Differ. Equ. 2015, 255 (2015)

33. Rihan, F.A., Abdel Rahman, D.H., Lakshmanan, S., Alkhajeh, A.S.: A time delay model of tumour-immune system interactions: global dynamics, parameter estimation, sensitivity analysis. Appl. Math. Comput. 232, 606-623 (2014)

34. Yang, R., Zhang, C., Zhang, Y.: A delayed diffusive predator-prey system with Michaelis-Menten type predator harvesting. Int. J. Bifurc. Chaos 28, 1850099 (2018)

35. Chinnathambi, R., Rihan, F.A.: Stability of fractional-order prey-predator system with time-delay and Monod-Haldane functional response. Nonlinear Dyn. 92, 1637-1648 (2018)

36. Song, P., Zhao, H., Zhang, X.: Dynamic analysis of a fractional order delayed predator-prey system with harvesting. Theory Biosci. 135, 59-72 (2016)

37. Wang, Z., Wang, X., Li, Y., Huang, X.: Stability and Hopf bifurcation of fractional-order complex-valued single neuron model with time delay. Int. J. Bifurc. Chaos 27, 945-955 (2018)

38. Yan, Y., Kou, C.: Stability analysis for a fractional differential model of HIV infection of CD4+ T-cells with time delay. Math. Comput. Simul. 82, 1572-1585 (2012)

39. Yuan, J., Zhao, L.Z., Huang, C.D., Xiao, M.: Novel results on bifurcation for a fractional-order complex-valued neural network with leakage delay. Physica A 514, 868-883 (2019)

40. Xu, C.J., Liao, M.X., Li, P.L., Guo, Y., Xiao, Q.M., Yuan, S.: Influence of multiple time delays on bifurcation of fractional-order neural networks. Appl. Math. Comput. 361, 565-582 (2019)

41. Pyragas, K.: Continuous control of chaos by self-controlling feedback. Phys. Lett. A 170, 421-428 (1992)

42. Zhao, H.T., Lin, Y.P., Dai, Y.X.: Bifurcation analysis and control of chaos for a hybrid ratio-dependent three species food chain. Appl. Math. Comput. 218, 1533-1546 (2011)

43. Huang, C., Song, X., Fang, B., Xiao, M., Cao, J.: Modeling, analysis and bifurcation control of a delayed fractional-order predator-prey model. Int. J. Bifurc. Chaos 28, 1850117 (2018)

44. Huang, C., Cao, J., Xiao, M., Alsaedi, A., Alsaadi, F.E.: Controlling bifurcation in a delayed fractional predator-prey system with incommensurate orders. Appl. Math. Comput. 293, 293-310 (2010)

45. Huang, C.D., Li, H., Cao, J.D.: A novel strategy of bifurcation control for a delayed fractional predator-prey model. Appl. Math. Comput. 347, 808-838 (2019)

46. Zhou, W.G., Huang, C.D., Xiao, M., Cao, J.D.: Hybrid tactics for bifurcation control in a fractional-order delayed predator-prey model. Physica A 515, 183-191 (2019)

47. Huang, C.D., Li, T.X., Cai, L.M., Cao, J.D.: Novel design for bifurcation control in a delayed fractional dual congestion model. Phys. Lett. A 383, 440-445 (2019)

48. Xu, C.J., Liao, M.X., Li, P.L.: Bifurcation control for a fractional-order competition model of Internet with delays. Nonlinear Dyn. 95(4), 3335-3356 (2019)

49. Xiao, M., Zheng, W.X., Lin, J.X., Jiang, G.P., Zhao, L.D., Cao, J.D.: Fractional-order PD control at Hopf bifurcations in delayed fractional-order small-world networks. J. Franklin Inst. 354, 7643-7667 (2017)

50. Wang, Q.D., Oksasoglu, A.: Strange attractors in periodically kicked Chua's circuit. Int. J. Bifurc. Chaos 15, 83-98 (2005) 
51. Xiao, M., Jiang, G., Cao, J., Zheng, W.: Local bifurcation analysis of a delayed fractional-order dynamic model of dual congestion control algorithms. IEEE/CAA J. Autom. Sin. 4, 361-369 (2017)

52. van den Driessche, P., Watmough, J.: Reproduction numbers and sub-threshold endemic equilibria for compartmental models of disease transmission. Math. Biosci. 180, 29-48 (2002)

53. Muth, E.J.: Transform Methods with Applications to Engineering and Operations Research. Prentice Hall, New Jersey (1977)

54. Deng, W.H., Li, C.P.: Synchronization of chaotic fractional Chen system. J. Phys. Soc. Jpn. 74, 1645-1648 (2005)

55. Bhalekar, S., Daftardar-Gejji, V.: A predictor-corrector scheme for solving nonlinear delay differential equations of fractional order. J. Fract. Calc. Appl. 4, 1-9 (2011)

Submit your manuscript to a SpringerOpen ${ }^{\circ}$ journal and benefit from:

- Convenient online submission

- Rigorous peer review

- Open access: articles freely available online

- High visibility within the field

- Retaining the copyright to your article

Submit your next manuscript at $\boldsymbol{\triangleright}$ springeropen.com 